\title{
Generation and control of helium-filled soap bubbles for PIV
}

\author{
David Engler Faleiros ${ }^{1,2}\left[\right.$ (D) Marthijn Tuinstra ${ }^{1} \cdot$ Andrea Sciacchitano $^{2} \cdot$ Fulvio Scarano ${ }^{2}$
}

Received: 14 September 2018 / Revised: 14 January 2019 / Accepted: 25 January 2019 / Published online: 14 February 2019

(c) The Author(s) 2019

\begin{abstract}
The operating regimes of an orifice-type helium-filled soap bubbles (HFSB) generator are investigated for several combinations of air, helium and soap flow rates to establish the properties of the production process and the resulting tracers. The geometrical properties of the bubbles, the production regimes and the production rates are studied with high-speed shadowgraphy. The results show that the bubble volume is directly proportional to the ratio of helium and air volume flow rates, and that the bubble production rate varies approximately linearly with the air flow rate. The bubble slip velocity is measured along the stagnation streamline ahead of a cylinder via particle image velocimetry (PIV), yielding the particle time response from which the neutral buoyancy condition for HFSB is inferred. The HFSB tracing capability approaches that of an ideal tracer (i.e., minimum slip and shortest response time) when the volume flow rate of helium is approximately one thousandfold the soap flow rate. This study provides guidelines for operating HFSB generation systems, intended for PIV experiments.
\end{abstract}

\section{Graphical abstract}
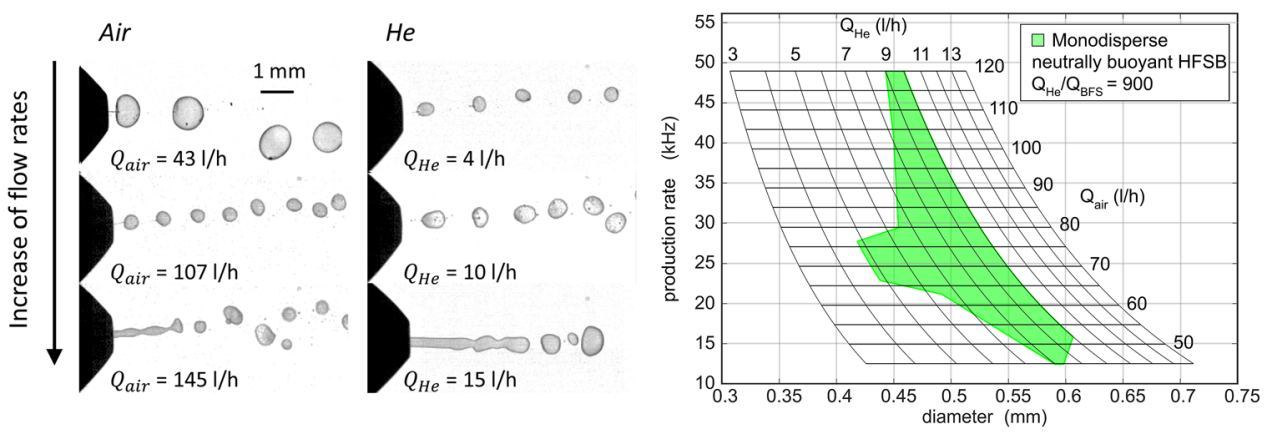

\section{Introduction}

The application of helium-filled soap bubbles (HFSB) for visualization of air flows dates back to the early work of Pounder (1956). In comparison to conventional tracers (e.g.,

Electronic supplementary material The online version of this article (https://doi.org/10.1007/s00348-019-2687-4) contains supplementary material, which is available to authorized users.

David Engler Faleiros

david.engler.faleiros@nlr.nl

1 Helicopters and Aeroacoustics Department, Netherlands Aerospace Centre (NLR), Emmeloord, The Netherlands

2 Faculty of Aerospace Engineering, Delft University of Technology, Delft, The Netherlands

fog or di-ethyl-hexyl-sebacat, DEHS), HFSB are much larger particles (typically from 0.3 to $5 \mathrm{~mm}$ ) that reflect 10,000 times more light (Caridi 2018). When illuminated with typical light sources used for particle image velocimetry (PIV), e.g., Nd:YAG and Nd:YLF lasers, they can still be observed from a distance of several meters. This makes them particularly suitable for large-scale PIV measurements, where, given the limitations of laser power, the finite scattering efficiency of tracer particles (dependent on particle size, refractive index, shape and orientation, polarization of the incident light and observation angle) usually limits the measured domain (Raffel et al. 2018).

The initial efforts to use HFSB for quantitative velocity measurements were discouraged due to difficulties of generating bubbles that could accurately follow the flow 
(Kerho and Bragg 1994). PIV measurements in low speed $(<1 \mathrm{~m} / \mathrm{s}$ ) convective flows using HFSB (Bosbach et al. 2009; Kühn et al. 2009) revived the idea of using these tracers for quantitative measurements. The feasibility of using HFSB for tomographic PIV was then investigated by Kühn et al. (2011) in a convective cell, showing calibration errors in the order of 0.1 pixels. The tracing fidelity of HFSB was later demonstrated for sub-millimeter bubbles in the steady flow upstream of a cylinder (Scarano et al. 2015), where the bubbles followed closely the streamlines obtained with fog droplets. A time response of $10 \mu$ s was inferred from the measured slip velocity within the deceleration field along the stagnation streamline. The tracing fidelity of HFSB has also been inquired within turbulent boundary layers (Faleiros et al. 2018) and in the core of concentrated vortices (Caridi et al. 2017). The good accuracy in the measurements of mean velocity and turbulence statistics found in the aforementioned works paves the way to the application of HFSB in a wide range of aerodynamic configurations, e.g., automotive, aerospace (Sciacchitano et al. 2018), wind energy (Caridi et al. 2016) and sports (Jux et al. 2018).

If bubble shape deformation is negligible (Sect. 2) and the bubble is "fully contaminated" (no slip at the surface), which is readily achieved through the addition of surfactants to the soap solution, the aerodynamic behavior of HFSB is closest to ideal when the tracer density equals that of the surrounding air (neutrally buoyant particle). Neutrally buoyant HFSB can be generated by carefully controlling the mass flows of helium and soap (Scarano et al. 2015). Additionally, it is worth noting that neutrally buoyant particles larger than the Kolmogorov scale were shown to sample turbulent motion similar to tracers, but filtered the turbulent motions happening at scales smaller than the particle diameter $(\mathrm{Xu}$ and Bodenschatz 2008).

HFSB are generated with an orifice-type bubble generator (Okuno et al. 1993; Bosbach et al. 2009, see Fig. 1) that is supplied with air, helium and bubble fluid solution (BFS) in a coaxial arrangement. The soap filament is extruded from the supply pipe by the helium and air flows. During this process, the film accelerates and reduces its thickness, forming an annular jet of soap filled with helium that is forced through the orifice. Depending on the production regime, the instability of the liquid film leads to a regular formation of helium-filled soap bubbles. The introduction of orifice-type bubble generators led to an increase in the production rate by an order of magnitude in comparison to a Pitot tube generator of similar geometry (Okuno et al. 1993). Additionally, the miniaturization of the orifice-type generators down to orifices of $1 \mathrm{~mm}$ diameter made it possible to achieve bubble production rates in the order of $10^{4}$ bubbles/s. Nevertheless, it remains a challenge to achieve adequate HFSB concentration for PIV experiments in wind tunnels.

Caridi et al. (2016) showed that a stream-tube in a wind tunnel could be seeded using a single generator by accumulating the bubbles in a reservoir for several seconds (the HFSB lifetime is of about 1-2 min as reported by Bosbach et al. 2009) and then rapidly releasing them. Large-scale PIV measurements over a wind turbine were demonstrated with this system. However, the efficiency of tracers transport and distribution in the stream was shown to be very low (the bubble concentration was one-third of the expected, which was attributed to bubbles bursting and colliding before reaching the test section) and, hence, the attention shifted towards the realization of rakes operating simultaneously tens or hundreds of generators, which need to be carefully engineered and manufactured, ensuring a consistent fluid supply to all generators and little variation in generator geometry. Otherwise, the tracer distribution will exhibit a wide dispersion of properties (diameter, mass, relative weight), resulting in large uncertainties of their aerodynamic behavior and of the associated measurement.

For a single generator, flow visualizations (Morias et al. 2016) show that bubble formation may occur in two regimes: bubbling and jetting. During bubbling, the bubbles are

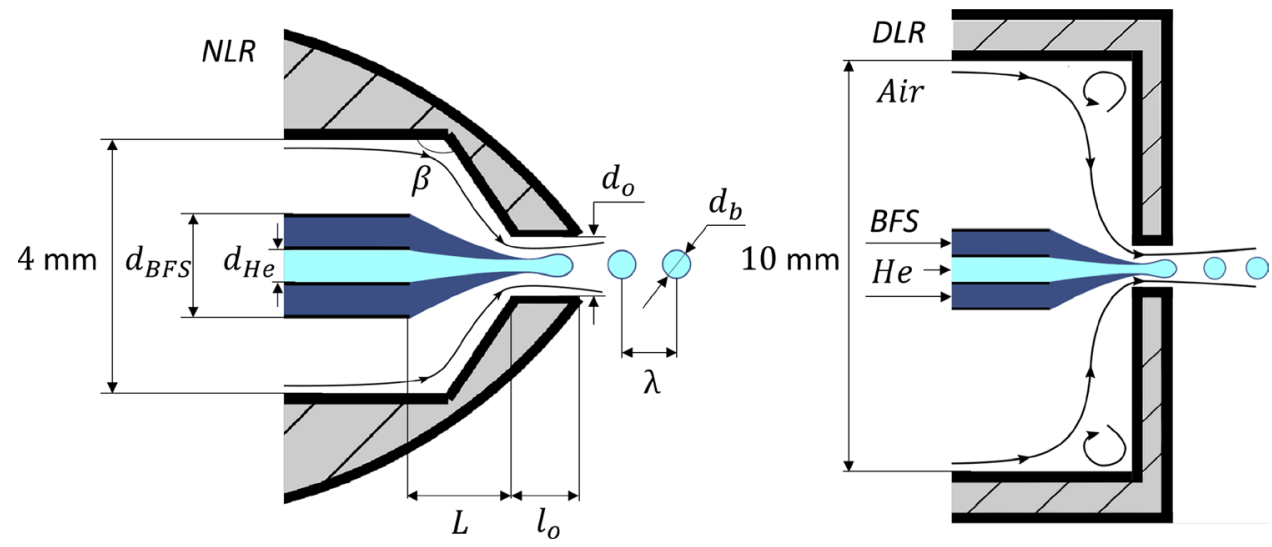

\begin{tabular}{ccc}
\hline Nozzle & NLR & DLR \\
\hline $\boldsymbol{\beta}$ & $125^{\circ}$ & $90^{\circ}$ \\
$\boldsymbol{d}_{\boldsymbol{B} F \boldsymbol{S}}$ & 2 & 2 \\
$\boldsymbol{d}_{\boldsymbol{h} \boldsymbol{e}}$ & 0.6 & 0.5 \\
$\boldsymbol{d}_{\boldsymbol{o}}$ & 1 & $\{0.75$ \\
$\boldsymbol{L}$ & 2 & 2 \\
$\boldsymbol{l}_{\boldsymbol{o}}$ & 1 & $1.5\}$ \\
\hline
\end{tabular}

Fig. 1 Schematic sectional view of the bubble generators used in the experiments (not to scale). Dimensions in $\mathrm{mm}$ 
formed within the bubble generator and present a rather stable and periodic formation. In the jetting regime, the cylindrical soap film extends beyond the nozzle exit and eventually breaks up into bubbles in a less regular process. Morias et al. (2016) and Faleiros et al. (2018) observed that in the bubbling regime the bubble diameter standard deviation was within $10 \%$ of the mean value. Morias et al. (2016) reported that by increasing the air flow rate the bubble size dispersion was approximately doubled, which was ascribed to the transition to the jetting regime. The HFSB time response was measured in the range from 10 to $40 \mu$ s irrespectively of their size dispersion; however, only a single case of bubbles produced in the jetting regime was included. Although of notably relevance, a detailed characterization of a bubble generator, exploring the limits of the operating regimes, is not available in the literature. The characterization of the production of HFSB and their properties is covered within the present study.

Guidelines for the production of neutrally buoyant HFSB were discussed in the literature to some extent for a nozzle of $1 \mathrm{~mm}$ orifice diameter. Bosbach et al. (2009), Scarano et al. (2015) and Morias et al. (2016) used a similar device and reported similar flow rates of BFS and helium $(5 \mathrm{ml} / \mathrm{h}$ and $5 \mathrm{l} / \mathrm{h}$, respectively), resulting in the production of neutrally buoyant HFSB. The flow rate of air was varied more broadly from $60 \mathrm{l} / \mathrm{h}$ (Bosbach et al. 2009) up to $160 \mathrm{l} / \mathrm{h}$ (Morias et al. 2016), although only a few conditions were tested. Some discrepancy is found in the reported bubble diameters and production rates. Bubble diameter observations vary from $0.23 \mathrm{~mm}$ (Bosbach et al. 2009) up to $0.55 \mathrm{~mm}$ (Morias et al. 2016; Faleiros et al. 2018). Reported bubble production rates varied from 50,000 bubbles per second (Morias et al. 2016; Caridi et al. 2016) up to 200,000 bubbles per second (Bosbach et al. 2009). Also in this regard, the literature lacks a systematic characterization of the relation between the fluid supply rates, the boundaries of the operation regime and the rates at which the bubbles are released. A recent study released at the time of writing this paper (Gibeau and Ghaemi 2018) presented measurements of HFSB properties for an 8-nozzle system of $0.8 \mathrm{~mm}$ orifice diameter. Bubble size and concentration were measured in the far field using the 8 nozzles simultaneously and neutrally buoyant bubbles were obtained using $8 \mathrm{ml} / \mathrm{h}$ of BFS and $10.8 \mathrm{l} / \mathrm{h}$ of helium per nozzle. They reported the bubble production rate to be dominated by the flow rates of soap and not significantly affected by the air flow rate, although the range of air flow rates tested was quite restricted (42-66 1/h). Furthermore, the authors reported that air flow rate did not influence significantly the size of the bubbles. Their approach of using 8 nozzles simultaneously brings extra uncertainty to the measurements, as there has been no verification that the nozzles were producing similar bubbles. Additionally, their investigation did not include sufficient testing conditions (e.g., larger flow rates of air) for a clear verification of the main influences of the input flow rates on the generated bubbles and the production regimes have not been included in the research.

Proper control of the generation of conventional micrometer oil particles is nowadays guaranteed, where a detailed study of atomizer nozzles (Kähler et al. 2002) delivered the relevant parameters affecting the particle size distribution. A thorough description of HFSB generation is hereby intended to permit the diffusion and standardization of these tracers for PIV. For a given generator geometry, the input parameters affecting the HFSB production are the fluid properties and volume flow rates of air, helium and BFS. The system output is monitored in terms of working regime (bubbling or jetting), average production rate, particle size, inferred weight and time response. The knowledge of such parameters is a prerequisite for a well-designed PIV experiment and estimation of the experimental uncertainty.

\section{Fundamental properties of bubbles as tracers}

\subsection{Size and production rate}

The bubble size can be determined through two different techniques. First, the diameter is obtained from the distance between glare points (often measured simultaneously with the time response). When a bubble is illuminated by a laser, with the bubble diameter being much larger (10-20 times) than the wavelength of the laser (Dehaeck et al. 2005), and recorded with a relatively large magnification so that the bubble size can be resolved, it is possible to distinguish the formation of two glare points. The orientation and distance of the glare points depend on the angle $\theta$ between the illumination and observation directions, on the thickness of the bubble, and on the refractive indexes of the bubble film, inner gas and surrounding medium. For a neutrally buoyant HFSB of about $0.5 \mathrm{~mm}$ diameter, the estimated thickness is of about $100 \mathrm{~nm}$ (Kerho and Bragg 1994) and the effects on light refraction can be neglected. Moreover, the refractive index of helium and air is approximately equal, $\eta_{\mathrm{He}} \approx \eta_{\mathrm{air}}$. Thus, in the simple case of negligible refraction of the laser light and for $\theta=90^{\circ}$ (planar PIV), the HFSB diameter is calculated as (Dehaeck et al. 2005):

$d_{\mathrm{b}}=\sqrt{2} d_{\mathrm{G}}$,

where $d_{\mathrm{G}}$ is the distance between the glare points.

The second method employs the shadow visualization of bubbles close to the generator exit to derive the diameter together with the establishment of production rate and working regime. Shortly after their release, the bubbles may 
not be spherical yet but rather of oval shape, being slightly elongated along the jet axis direction. The bubble size is thus calculated by fitting an ellipse to the edge of the bubble shadow, and assuming it to be an ellipsoid in three-dimensional space. Assuming axisymmetric flow, the two smaller semi-axes are equal and the bubble volume is calculated as:

$V_{\mathrm{b}}=\frac{4}{3} \pi a b^{2}$,

in which $a$ and $b$ are, respectively, the semi-major and semiminor axes of the bubble elliptical cross-section. The bubble diameter is then estimated for the reference condition of the bubble reaching spherical shape, which is valid further away from the nozzle exit:

$d_{\mathrm{b}}=\left(\frac{6}{\pi} V_{\mathrm{b}}\right)^{\frac{1}{3}}$.

Assuming constant flow rate of helium, no helium leakage during the bubble formation, and neglecting the soap film thickness, the volume of the bubble can also be determined by integrating the volume flow rate of helium $Q_{\mathrm{He}}$ during the bubble formation time $(1 / f)$ :

$V_{\mathrm{b}}=\frac{Q_{\mathrm{He}}}{f}$.

The bubble production rate $f$ is defined by the number of bubbles crossing a given target per the unit time. The production rate can also be estimated as the ratio of bubble velocity and separation length $\lambda$ (Fig. 1) right after their formation:

$f=\frac{u_{\mathrm{b}}}{\lambda}$.

The parameters $f, u_{\mathrm{p}}$ and $\lambda$ are measured independently by counting and tracking the bubbles and measuring the distance between them via high-speed flow visualization.

\subsection{Time response and neutral buoyancy}

In the ideal case of no soap spillage or helium leakage during the bubble formation process, the following relation can be obtained imposing mass conservation

$f V_{\mathrm{b}} \rho_{\mathrm{b}}=Q_{\mathrm{He}} \rho_{\mathrm{He}}+Q_{\mathrm{BFS}} \rho_{\mathrm{BFS}}$,

where $\rho_{\mathrm{He}}, \rho_{\mathrm{BFS}}$ and $\rho_{\mathrm{b}}$ are the helium, soap and bubble densities, respectively. Substituting (4) into (6) yields:

$\rho_{\mathrm{b}}=\rho_{\mathrm{He}}+\frac{Q_{\mathrm{BFS}}}{Q_{\mathrm{He}}} \rho_{\mathrm{BFS}}$.

Thus, the neutral buoyancy condition $\left(\rho_{\mathrm{b}}=\rho_{\text {air }}\right)$ at normal temperature and pressure is satisfied when $Q_{\mathrm{He}} / Q_{\mathrm{BFS}} \cong 1080$.
The production of neutrally buoyant bubbles is a requirement for an accurate PIV measurement. When shape deformation is negligible, a bubble experiences the same drag of a solid spherical particle, and its time response to fluid acceleration is linearly related to the difference in density between the bubble and the surrounding fluid (see Fig. 3 for an illustration of particle time response effects). In the Stokes regime, the time response of a particle of diameter $d_{\mathrm{p}}$ in a fluid of density $\rho$ and kinematic viscosity $v$ is (Adrian and Westerweel 2011)

$\tau_{\mathrm{p}}=\frac{\bar{\rho} d_{\mathrm{p}}^{2}}{18 v \phi}$

in which $\bar{\rho}=\left(\rho_{\mathrm{p}}-\rho\right) / \rho$ and $\phi$ is a factor dependent on the particle Reynolds number (Clift et al. 1978). An empirical expression for $\phi$ (Schiller and Naumann 1933, as cited in Clift et al. 1978) in the case $R e_{\mathrm{p}}<800$ is

$\phi=1+0.15 R e_{\mathrm{p}}^{0.687}, \quad R e_{\mathrm{p}}=\frac{\left(u_{\mathrm{p}}-u\right) d_{\mathrm{p}}}{v}$,

where $u_{\mathrm{p}}$ and $u$ are the magnitude velocity of the particle and surrounding fluid, respectively. Other expressions for $\phi$ for different $R e_{\mathrm{p}}$ ranges can be found in Clift et al. (1978). Furthermore, it is noted that the time response of a finite particle is dependent on the fluid acceleration due to the non-linear $\phi$ term.

The time response of the tracers can also be inferred as the ratio of slip velocity and particle acceleration, which in the streamwise direction is

$\tau_{\mathrm{p}}=\frac{\left|u-u_{\mathrm{p}}\right|}{\left|u_{\mathrm{p}} \frac{\partial u_{\mathrm{p}}}{\partial x}\right|}$,

and it may be normalized by selecting a proper flow time scale, resulting in the so-called Stokes number:

$S_{\mathrm{tk}}=\frac{\tau_{\mathrm{p}}}{\tau_{\mathrm{f}}}$.

The technique used to measure the aforementioned properties is based on the dynamic forces that a spherical particle undergoes (Mei 1996) during deceleration in the steady laminar flow ahead of a circular cylinder (Scarano et al. 2015). In this case, the the time scale of the flow may be defined as the ratio of the cylinder diameter and the free stream velocity $\tau_{\mathrm{f}}=D / U_{\infty}$. This approach has been adopted in several studies (Morias et al. 2016; Faleiros et al. 2018) producing estimations of particle response times consistent with the characteristics of the bubbles. The HFSB velocity and acceleration are obtained through particle tracking velocimetry (PTV) and the reference velocity field required for computing the slip velocity is measured with PIV, using 
micrometric DEHS droplets as tracers. With the bubble diameter measured from the glare points, the bubble density is then estimated from Eq. (8).

\subsection{Bubble shape}

The interaction between fluid-dynamic stresses acting on the bubble surface (causing deformation) and the counteracting surface tension stresses (resisting deformation) is responsible for changes in the bubble shape. When these changes are small, the bubble takes the shape of a spheroid and the deviation from spherical shape (eccentricity) is quantified by its aspect ratio, i.e., the ratio between the diameter along the axis of symmetry and the diameter about the axis of symmetry $\left(E=d_{\|} / d_{\perp}\right)$. For small $R e_{\mathrm{p}}$ (1 to 10,000$)$, the deformation is governed by the Weber number (Loth 2008):

$W e_{\mathrm{p}}=\frac{\rho\left(u_{\mathrm{b}}-u\right)^{2} d_{\mathrm{b}}}{\sigma}$,

where $\sigma$ is the surface tension between the bubble surface and the surrounding fluid. If $W e_{\mathrm{p}} \ll 1$, particles tend rapidly to spherical geometry. When $W e_{\mathrm{p}} \sim 1$, the bubbles suffer moderate deformation. If $W e_{\mathrm{p}} \gg 1$, the bubble may deviate significantly from a sphere. The theoretical onset of ellipsoidal condition $(E \leq 0.9)$ of a clean bubble occurs at $W e_{\mathrm{p}}=0.64$ (Taylor and Acrivos 1964, as cited by Loth 2008).

\section{Experimental setup and procedures}

\subsection{Instrumentation}

The main bubble generator employed in the experiments is an NLR design (Fig. 1, left) of $1 \mathrm{~mm}$ orifice diameter, which is a CNC-manufactured generator based on the $3 \mathrm{D}$ printed HFSB-GEN-V11 generator developed at TU Delft. Another bubble generator geometry (Fig. 1, right) based on a DLR design (Bosbach et al. 2009) was also tested for comparison. The scaling properties of bubble generation were investigated adopting three different values of the orifice diameter $d_{\mathrm{o}}$, namely $0.75,1$ and $1.5 \mathrm{~mm}$. The main difference of the NLR design in comparison to that of DLR consists in a conical contraction region upstream of the orifice $\left(\beta>90^{\circ}\right)$. This modification is intended to avoid flow separation at the junction between the cylindrical part and the end wall, where residues of recirculating soap fluid accumulate under specific conditions.

The gases and soap mass flows are set, respectively, using El-Flow Select and Mini Cori Flow mass flow controllers from Bronkhorst. The bubble fluid solution used is the SAI 1035 from Sage Action, Inc. The properties of the fluids used are given in Table 1. The images were recorded with a LaVision HighSpeedStar 5 CMOS camera $(1024 \times 1024$ pixels, 12 bits, $20 \mu \mathrm{m}$ pixel pitch). The laser used during PIV measurements is a diode pumped Litron Nd:YLF LDY304 $(2 \times 30 \mathrm{~mJ} /$ pulse at $1 \mathrm{kHz})$.

\subsection{PIV/PTV}

HFSB size and time response were measured in the deceleration region in front of a cylinder using PIV with DEHS particles as reference flow field and PTV with HFSB to obtain slip velocity and particle deceleration. Measurements were performed in the small anechoic wind tunnel KAT of The Netherlands Aerospace Centre (NLR) using a cylinder of $50 \mathrm{~mm}$ diameter, spanning the $38 \times 51 \mathrm{~cm}^{2}$ test-section. HFSB were generated using the NLR nozzle. The wind tunnel speed was set at $30 \mathrm{~m} / \mathrm{s}$. The resulting deceleration ahead of the cylinder is in the order of $7000 \mathrm{~m} / \mathrm{s}^{2}(700 \mathrm{~g})$. A total of 35 combinations of volume flow rates of air $(65-160 \mathrm{l} / \mathrm{h})$, helium (4-21 $\mathrm{l} / \mathrm{h})$ and soap $(3.5-11 \mathrm{ml} / \mathrm{h})$ were tested.

Planar PIV measurements were performed in frame straddling mode at $50 \mathrm{~Hz}$ on a laser sheet thickness of $3 \mathrm{~mm}$. A total of 3500 images were acquired. Image analysis by crosscorrelation was performed with the DaVis 8.4 software from LaVision, which provided an accurate reference for the velocity field of the air flow. For the PTV measurements, the laser was operated at $20 \mathrm{kHz}$ and 10,000 multi-exposure images per condition were recorded at a rate of $100 \mathrm{~Hz}$ with time exposure of $0.01 \mathrm{~s}$. The camera sensor was cropped to $512 \times 320$ pixels, resulting in a field of view of $23 \times 14 \mathrm{~mm}^{2}$. The lens focal length was $200 \mathrm{~mm}$, resulting in a magnification of 0.44 and an image resolution of $22.15 \mathrm{pixel} / \mathrm{mm}$. The bubbles were imaged in about 10 pixels, allowing the pair of glare points to be resolved (Sect. 2.1). Those were found as local maxima in the image. Their center position was obtained with subpixel accuracy by fitting a Gaussian function to their intensity distribution. The coupling of glare points, and therefore the bubble identification, was quite straightforward as the image particle concentration was sufficient low. For that, the distance and orientation between the glare points were used. The minimum distance between the glare points considered in the processing was of 3 pixels $(0.13 \mathrm{~mm})$. The size of the bubbles was then retrieved from the distance between the glare points

Table 1 Fluid properties at $20{ }^{\circ} \mathrm{C}$ and $1 \mathrm{~atm}$

\begin{tabular}{llll}
\hline Fluid & $\begin{array}{l}\text { Density, } \rho(\mathrm{kg} / \\
\left.\mathrm{m}^{3}\right)\end{array}$ & $\begin{array}{l}\text { Dynamic viscosity, } \\
\mu(\mathrm{Pa} \mathrm{s})\end{array}$ & $\begin{array}{l}\text { Surface } \\
\text { tension, } \sigma \\
(\mathrm{mN} / \mathrm{m})\end{array}$ \\
\hline Air & 1.20 & $1.8 \times 10^{-5}$ & - \\
Helium & 0.17 & $2.0 \times 10^{-5}$ & - \\
BFS & 1124 & $8.0 \times 10^{-3}$ & 27.5 \\
\hline
\end{tabular}


(Sect. 2.1). The bubble centroid was obtained as the midpoint between the glare points. Polynomial fitting was then applied to the center of the particles along the trajectories captured in the multi-exposure images rendering particle velocity and acceleration. For more details on the data processing, the reader is referred to Faleiros et al. (2018).

The uncertainty of the measured diameter was verified by checking the variation of bubble diameter along bubble trajectories, where physical variations of bubble size are negligible and occur mainly due to the method itself. This has been done for each tested condition, using 1000 trajectories with more than ten bubbles each. The diameter standard deviation relative to the mean along a trajectory was larger for smaller bubbles, and consisted of $2 \%$ for $\left\langle d_{\mathrm{b}}\right\rangle=0.3 \mathrm{~mm}$, decreasing as the mean diameter increased, down to $1.2 \%$ for $\left\langle d_{\mathrm{b}}\right\rangle=0.8 \mathrm{~mm}$. The uncertainty of the mean bubble diameter of all samples is even smaller. Assuming a normal distribution, the uncertainty may be calculated as $3 \sigma_{\mathrm{d}} / \sqrt{N}$, where $\sigma_{\mathrm{d}}$ is the diameter standard deviation, $N$ is the number of samples and the factor three means it is computed at $99.7 \%$ confidence level. This is less than $0.3 \%$ of the mean bubble diameter for all tested conditions.

\subsection{Visualization technique}

The production regimes (mode of bubble production) and the production rate were investigated via shadow visualization of the bubbles at the exit of the generator. Moreover, an investigation of the HFSB size was realized for a broader range of fluid conditions than in the PIV experiment. The measurements were performed using the high-speed camera (camera lens of $200 \mathrm{~mm}$ focal length) placed perpendicular to the bubble stream and opposite to a continuous LED light source (Fig. 2). Recordings at $70 \mathrm{kHz}$ frame rate with an exposure time of $1 \mu$ s provided images of HFSB resolved in time and space. The image sensor was cropped to $320 \times 192$ pixels with a magnification factor of 0.78 , resulting in a FoV of $8 \times 5$ $\mathrm{mm}^{2}$. The image resolution was $40 \mathrm{pixel} / \mathrm{mm}$. The volume flow rates of helium $Q_{\mathrm{He}}(4-17 \mathrm{l} / \mathrm{h})$ and air $Q_{\text {air }}(20-180 \mathrm{l} / \mathrm{h})$ were varied to cover the working regime for five BFS flow rates $Q_{\mathrm{BFS}}(3.5-11 \mathrm{ml} / \mathrm{h})$, resulting in several visual observations and 530 recorded conditions for further analysis. Most of the results presented (310 recordings) are based on the NLR bubble generator. The DLR generator is only included in the bubble size and production rate analysis (Sect. 7). Each recording comprises ten uncorrelated cycles of 500 images realized with a time interval of $200 \mathrm{~ms}$ from each other.

A detection and tracking in-house algorithm renders the bubble size (Sect. 2.1) and the bubble velocity and counts the number of bubbles produced during each cycle. The bubbles are tracked from the nozzle exit until they leave the frame. The production rate in a single cycle is then calculated as:

$f_{\text {cycle }}=\frac{n_{\mathrm{b}}}{\left(N_{\mathrm{i}}-1\right)} f_{\mathrm{c}}$,

in which $n_{\mathrm{b}}$ is the number of generated bubbles in one cycle, $N_{\mathrm{i}}$ is the number of images per cycle and $f_{\mathrm{c}}$ is the camera acquisition frequency. The bubble production rate $f$ is then defined as the average of $f_{\text {cycle }}$ of all recorded cycles.

\section{HFSB time response and density}

The effect of particle time response can be observed qualitatively through the streamlines in front of the cylinder (Fig. 3). The black streamlines show the reference measurements obtained using DEHS $\left(\left\langle\tau_{\mathrm{p}}\right\rangle=2 \mu\right.$ s according to Ragni et al. 2011). Nearly neutrally buoyant HFSB $(\langle\bar{\rho}\rangle$ $\left.=0.05,\left\langle\tau_{\mathrm{p}}\right\rangle=20 \mu \mathrm{s}\right)$ follow closely the reference streamlines. Heavier-than-air bubbles $\left(\langle\bar{\rho}\rangle=0.5,\left\langle\tau_{\mathrm{p}}\right\rangle=130 \mu \mathrm{s}\right)$ take longer to respond to the deceleration caused by the pressure gradient due to their inertia and, therefore, the particles approach closer to the cylinder before turning sideways. Lighter-thanair bubbles $\left(\langle\bar{\rho}\rangle=-0.2,\left\langle\tau_{\mathrm{p}}\right\rangle=-90 \mu \mathrm{s}\right.$, negative sign indicating lighter than air) respond in a shorter distance (to a lower threshold of pressure drag) than air itself, as a result of their lower inertia force.

The bubble slip velocity, acceleration and diameter are measured for each bubble crossing the dashed area shown in Fig. 3. The $W e_{\mathrm{p}}$ is at least one order of magnitude smaller (Fig. 4) than the expected onset of ellipsoidal condition ( $E \leq 0.9$, Sect. 2.3); thus, the bubble deformation is considered negligible. The time response is obtained from (10). The theoretical estimation for HFSB density-Eq. (7) - and the calculated ratio $Q_{\mathrm{He}} / Q_{\mathrm{BFS}}$ leading to neutrally buoyant bubbles are then verified by estimating the bubble density from Eq. (8).

The dependency of the normalized density difference $\bar{\rho}$ upon the ratio $Q_{\mathrm{He}} / Q_{\mathrm{BFS}}$ is shown in Fig. 5 (left). The curve fit $\left(\langle\bar{\rho}\rangle=-0.434+392.2\left(Q_{\mathrm{He}} / Q_{\mathrm{BFS}}\right)^{-1}\right)$ has a

Fig. 2 Schematic illustration of the experimental setup for bubbles shadow visualization

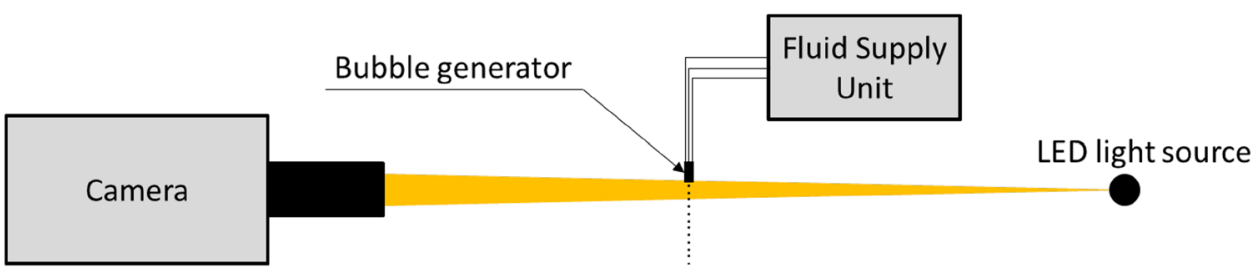


Fig. 3 Flow field ahead of the circular cylinder obtained from PIV measurements (velocity contours and solid black lines as streamlines). Trajectories followed by neutrally buoyant (red), heavier-than-air (green) and lighter-than-air (blue) HFSB tracers. Dashed region is used for the calculation of HFSB time response

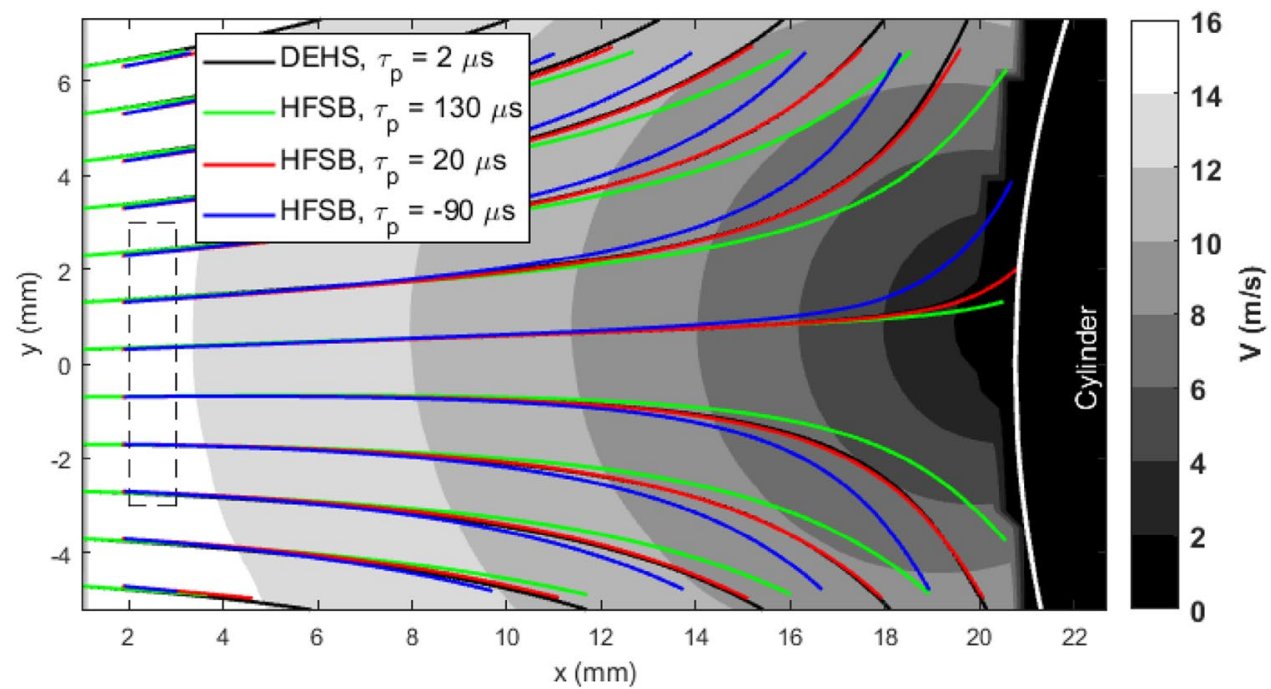

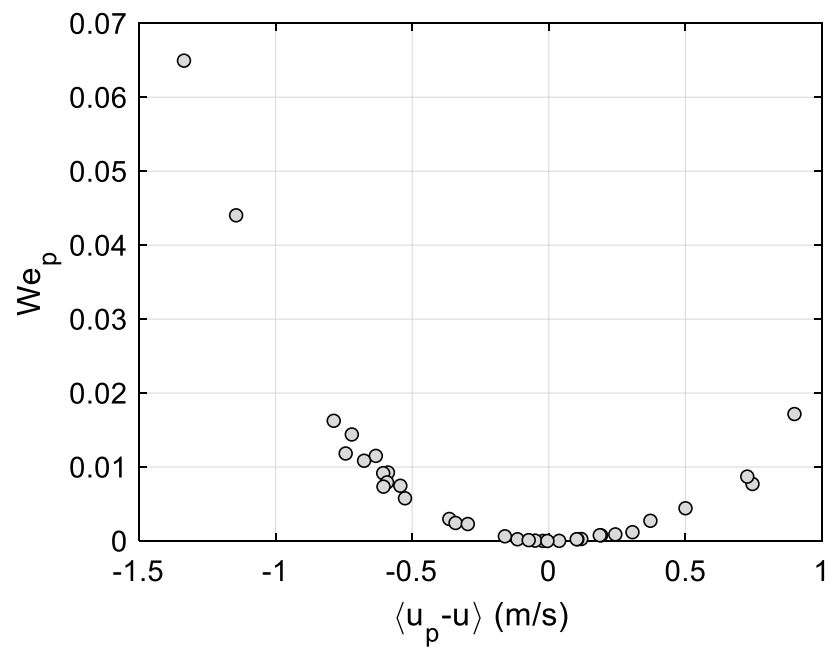

Fig. 4 HFSB Weber numbers for the tested conditions

similar shape as the theoretical curve. The point where it crosses the neutrally buoyant line $(\bar{\rho}=0)$ gives the ratio of helium and soap volume flow rates resulting in HFSB with shortest time response, that is $Q_{\mathrm{He}} / Q_{\mathrm{BFS}}=900$. The standard deviation of the neutral buoyancy ratio is obtained by a Monte Carlo simulation. For each combination of mass flows that has been tested (35 in total), there is a distribution of $\bar{\rho}$ values of all the recorded bubbles corresponding to a single $Q_{\mathrm{He}} / Q_{\mathrm{BFS}}$ ratio. In every iteration of the Monte Carlo simulation, a single $\bar{\rho}$ value is randomly selected from each distribution, forming a group of $35\left(\bar{\rho}, Q_{\mathrm{He}} / Q_{\mathrm{BFS}}\right)$ points. A least-square fit is then applied to the points and the ratio of neutral buoyancy $\left(Q_{\mathrm{He}} / Q_{\mathrm{BFS}}\right.$ for $\left.\bar{\rho}=0\right)$ of that iteration is found. This procedure was repeated 10,000 times and the standard deviation of the optimal ratio was calculated. From the simulation, it is then obtained that the neutral buoyancy ratio is $Q_{\mathrm{He}} / Q_{\mathrm{BFS}}=900 \pm 50$ (interval of \pm one standard deviation).

The more gradual slope of the empirical curve of $\langle\bar{\rho}\rangle$ may be ascribed to the non-negligible effect of the addedmass force (Faleiros et al. 2018) causing the theoretical estimate of time response, and consequently of density, to be slightly overestimated, i.e., particles follow the flow better than predicted by Stokes flow theory. Nevertheless, this would not change the neutral buoyancy condition $\left(\bar{\rho}=0, \tau_{\mathrm{p}}=0\right)$. This is attributed to spillage of soap in the bubble formation process, which causes the neutral buoyancy condition to be achieved at slightly lower helium flow rates. It is, therefore, advised to introduce a correction to the theoretical density curve (7) to account for the violation of soap mass conservation

$\rho_{\mathrm{b}}=\rho_{\mathrm{He}}+K \frac{Q_{\mathrm{BFS}}}{Q_{\mathrm{He}}} \rho_{\mathrm{BFS}}, \quad K=900\left(\rho_{\mathrm{air}}-\rho_{\mathrm{He}}\right) / \rho_{\mathrm{BFS}}$,

in which $\rho_{\mathrm{b}}=\rho_{\text {air }}$ when $Q_{\mathrm{He}} / Q_{\mathrm{BFS}}=900$. The factor $K \cong 0.84$ is interpreted as a correction for the soap that does not end up into bubbles, but is discarded via tiny droplets naturally occurring during bubble production.

According to (7) and (8), the HFSB time response should vary linearly with $Q_{\mathrm{BFS}} / Q_{\mathrm{He}}$. This assumption is verified in Fig. 5 (right). The Pearson correlation coefficient $r_{x, y}=\operatorname{cov}(x, y) / \sigma_{x} \sigma_{y}$, where $\operatorname{cov}(x, y)$ is the covariance and $\sigma$ is the standard deviation, shows a linear correlation of 0.88 . Deviations from a linear dependence are observed, especially for large $\left\langle\tau_{\mathrm{p}}\right\rangle$. Particles with longer relaxation time will in general present larger particle Reynolds numbers and, consequently, the correction factor $\phi\left(R e_{\mathrm{p}}\right)$ will be more significant, affecting the linearity between $\tau_{\mathrm{p}}$ and $\bar{\rho}$. Moreover, 


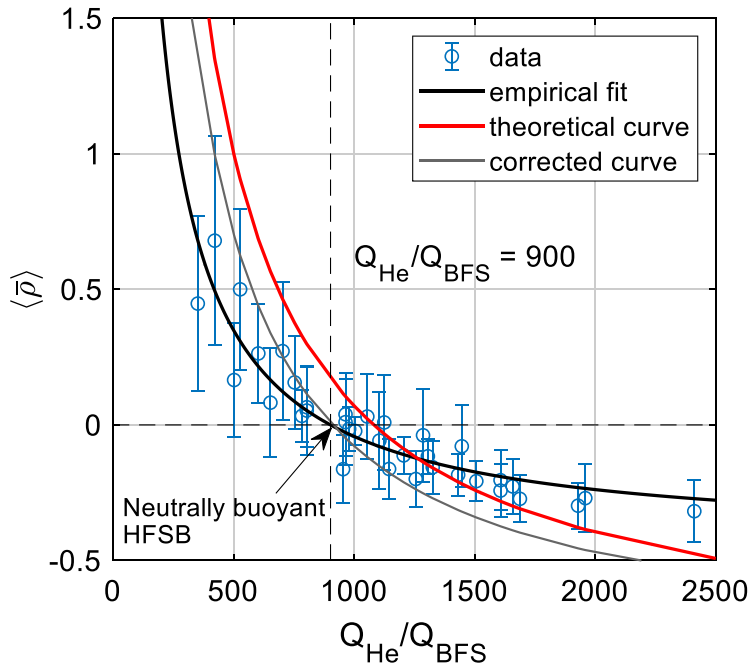

Fig. 5 HFSB time response and density difference as a function of helium and soap flow rates, measured using the NLR bubble generator. Left: normalized HFSB density difference. The error bars represent one standard deviation. Right: HFSB time response. Linear

variations in the particle diameter may also affect the proportionality between the density and time response.

The relation between particle time response and diameter (Fig. 6), which have been both simultaneously measured, is shown for different HFSB densities. The measured $\left\langle\tau_{\mathrm{p}}\right\rangle$ presents a negative Pearson correlation of -0.75 with $\left\langle d_{\mathrm{p}}\right\rangle$. This is a consequence of the fact that normally lighter-than-air bubbles are large, whereas heavier-than-air bubbles are small. This negative correlation between $\langle\bar{\rho}\rangle$ and $\left\langle d_{\mathrm{p}}\right\rangle$ of -0.65 dominates, therefore, the trend observed in Fig. 6. The dependency between diameter and time response must,

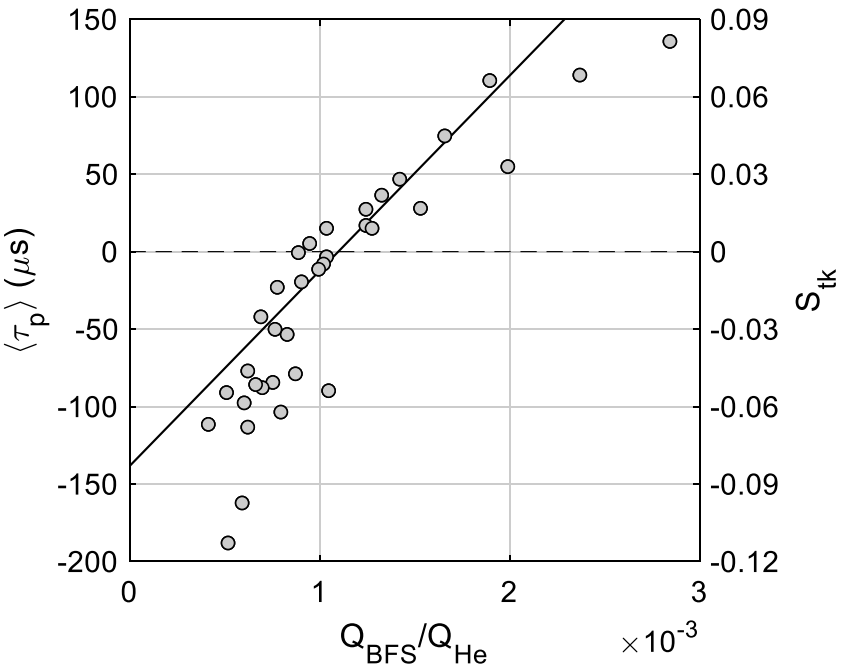

fit of $\left\langle\tau_{\mathrm{p}}\right\rangle$ is enforced to include the neutrally buoyant condition $\tau_{\mathrm{p}}$ $\left(Q_{\mathrm{He}} / Q_{\mathrm{BFS}}=900\right)=0$, emphasizing the deviation from the predicted linear relation between $\tau_{\mathrm{p}}$ and $\bar{\rho}$ given by Eq. (8)

therefore, be analyzed for different density values. To avoid biasing the results, instead of using the density values that were estimated from Eq. (8), which would enforce $\tau_{\mathrm{p}}$ to be proportional to $\bar{\rho}$, the density is estimated from (14). As reference, theoretical curves of time response $\tau_{\mathrm{t}}$ obtained from (8) are included for fixed density values. To calculate the correction factor $\phi\left(R e_{\mathrm{p}}\right)$ that was used in the theoretical curves, a constant particle deceleration of $7000 \mathrm{~m} / \mathrm{s}^{2}$ is assumed and the particle slip velocity is estimated as $\left(u_{\mathrm{p}}-u\right) \sim a_{\mathrm{p}} \tau_{\mathrm{p}}$. It is noted that $\phi\left(R e_{\mathrm{p}}\right)$ influences the dependence of $\tau_{\mathrm{p}}$ on $d_{\mathrm{p}}$, showing an approximately linear relation for $d_{\mathrm{p}}>0.1 \mathrm{~mm}$. From
Fig. 6 HFSB time response in relation to bubble diameter

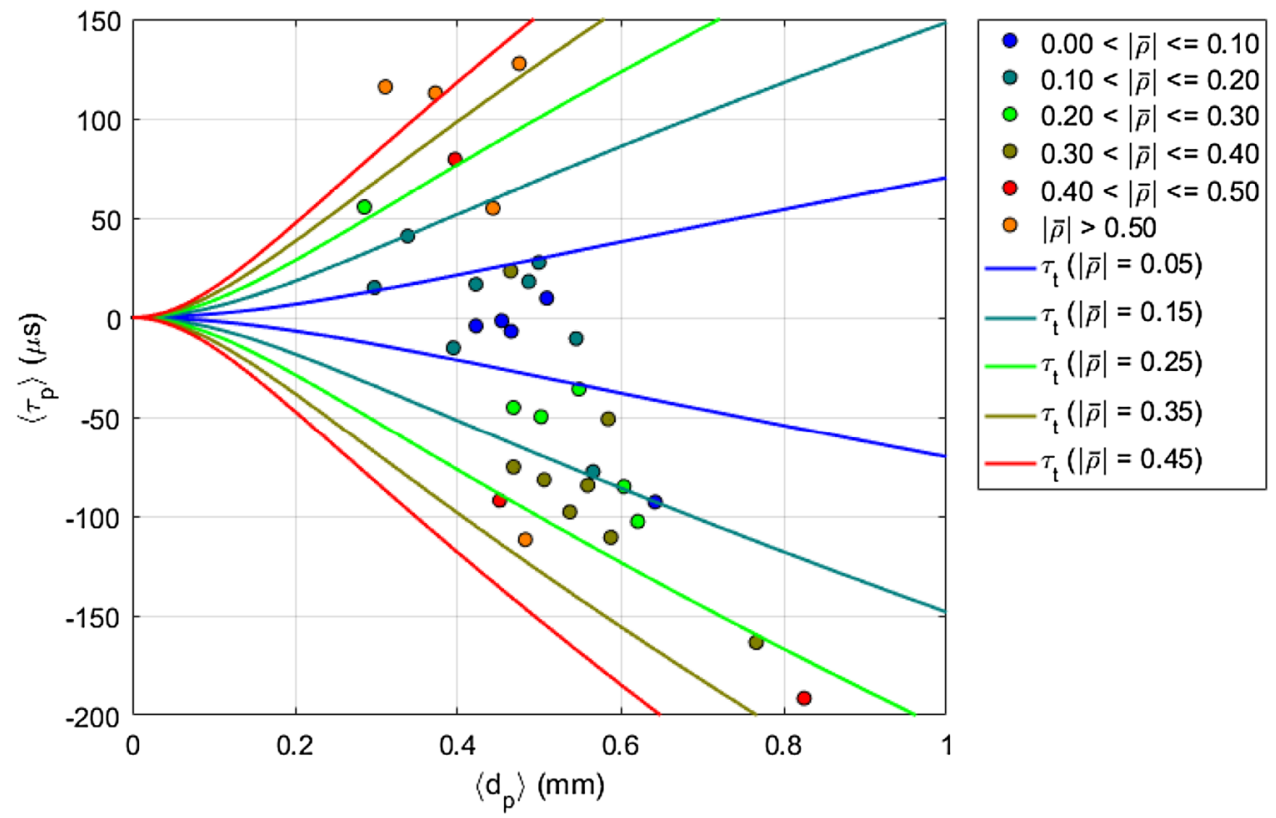


the equation of the correction factor (9) and still under the assumption that $\left(u_{\mathrm{p}}-u\right) \sim a_{\mathrm{p}} \tau_{\mathrm{p}}$, one can see that for small $d_{\mathrm{p}}$, $\tau_{\mathrm{p}} \sim d_{\mathrm{p}}^{2}$, while for large $d_{\mathrm{p}}, \tau_{\mathrm{p}} \sim d_{\mathrm{p}}^{0.78}$. Although there are not enough data points of similar density to verify such relations, it is observed that the theoretical curves mostly overestimate (by a factor 2 approximately) the measured time response values providing safe limits for the expected boundaries of time response. As mentioned before, the measured time response is most likely smaller than that predicted by steady Stokes flow theory due to the added mass force effect.

\section{Production regimes}

\subsection{Shadow visualization of production regimes}

For a given generator geometry and fluid properties, the combination of the fluids volume flow rates determines the type of bubbles that are produced. A qualitative classification of the regimes of bubble production (Fig. 7) facilitates the discussion of the different phenomena involved in bubble formation. The main distinction is based on whether the bubble forms inside or outside of the nozzle. Exterior bubble formation is here defined as jetting, where the bubble is formed and detaches from the jet at least one bubble diameter downstream of the nozzle exit. If the bubble is formed closer to the nozzle (normally within the orifice), the production regime is defined as bubbling. Other distinctions are based on whether the production of bubbles is monodisperse, i.e., periodic formation of bubbles with constant size or visibly polydisperse. Monodisperse generation of bubbles is mainly obtained in the bubbling regime, however, with a few exceptions. A specific regime is recognized when alternated production of two distinct populations of monodisperse bubbles coexists (double bubbling). Merging of bubbles has also been observed during double bubbling or polydisperse production. Another observed phenomenon
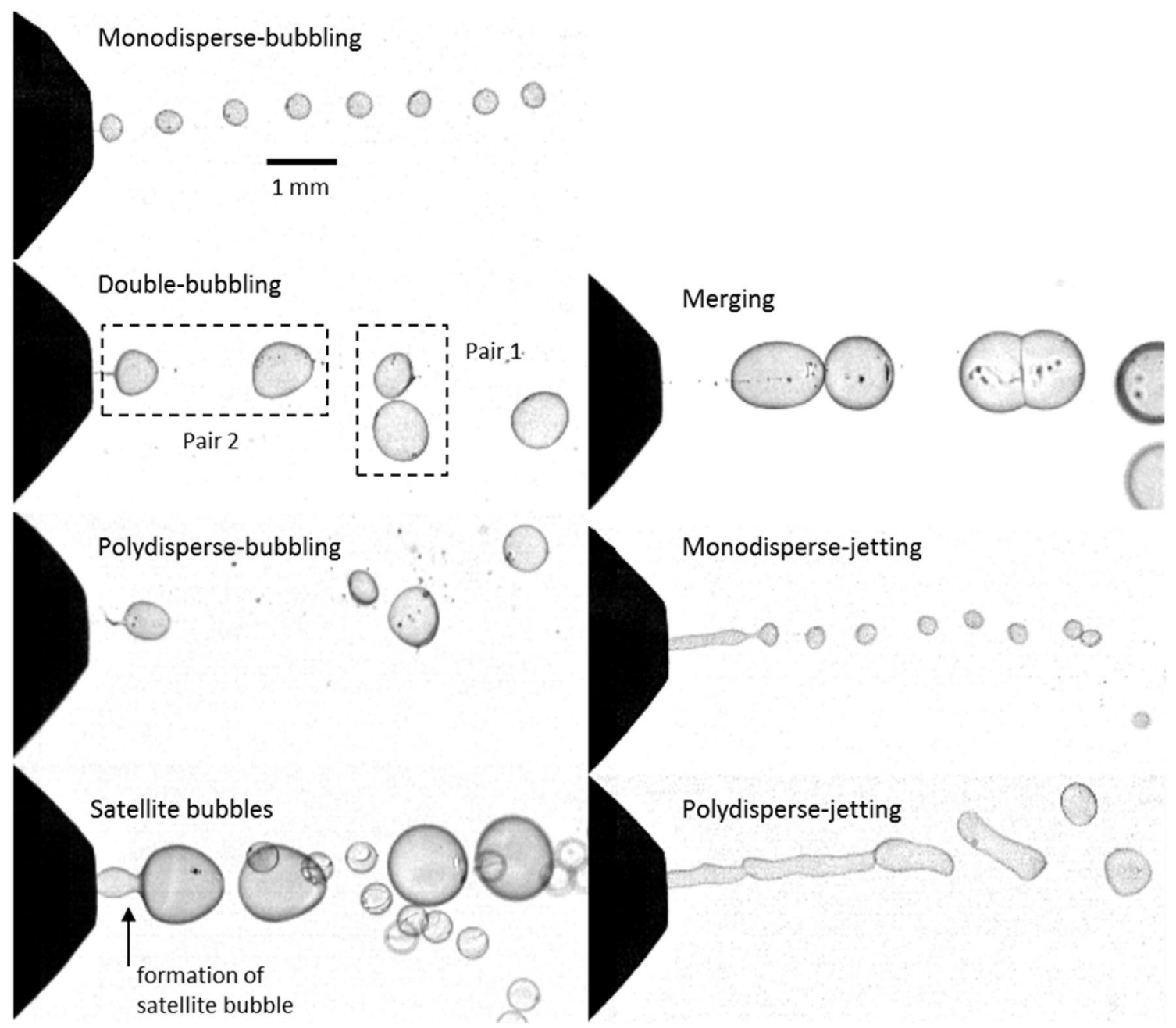

Fig. 7 Shadow visualization of production regimes and their classification 
is that in some cases a satellite bubble is formed in between the detachment point of bubble formation and the envelope of helium and soap. Although satellite bubbles are present also in other regimes, in most of the cases they are significantly smaller than the main bubbles and can be neglected. For ease of communication, double bubbling, polydisperse bubbling, large satellite bubbles and merging will be altogether referred to as polydisperse bubbling, and monodisperse periodic bubble production will be referred to simply as bubbling. Furthermore, although monodisperse bubble formation may be occasionally obtained in the jetting regime for a small range of flow rates, such condition is often unstable and turns into irregular (polydisperse) production by a slight change of flow rates. Thus, prioritizing the discussion from the perspective of PIV utilization, most attention is devoted to the condition that yields stable and monodisperse bubbles, i.e., the bubbling regime.

Guidelines for avoiding jetting may be derived from observations of its occurrence in relation to the imposed flow rates. The effects of volume flow rate variation are observed for independent increase of air, helium and soap volume flow rate, while keeping the flow rates of the other two fluids constant (Fig. 8). Observation of air flow rate variation shows that relatively low volume flow rates result in the generation of large bubbles in the bubbling regime. By increasing the air flow rate, the bubbles start to reduce in size and, consequently, the distance between bubbles reduces, suggesting an increase in the production rate. At $Q_{\text {air }}=1291 / \mathrm{h}$, a jet extending about two bubble diameters away from the nozzle is formed, yet a rather monodisperse bubble size distribution is observed (monodisperse-jetting). As the volume flow rate of air is increased to $Q_{\text {air }}=145 \mathrm{l} / \mathrm{h}$, the jet extends to about four bubble diameters downstream of the nozzle and the soap film breaks up irregularly, yielding a polydisperse distribution of bubbles. Low volume flow rates of helium also tend to yield bubbling regime. Differently from the effect of varying air flow rate, the bubble size increases by increasing the helium flow rate and no clear change in the distance between the bubbles is observed. The transition to jetting by increasing the volume flow rate to $Q_{\mathrm{He}}=11.8 \mathrm{l} / \mathrm{h}$ is more abrupt in this case and a longer jet of about six bubble diameters is observed. It is noted that the smaller jet length in the snapshot shown for $Q_{\mathrm{He}}=15 \mathrm{l} / \mathrm{h}$ relative to the case of $Q_{\mathrm{He}}=11.8 \mathrm{l} / \mathrm{h}$ should not be interpreted as a reduction in the jet size, as it is an instantaneous representation of a very dynamical and unsteady state. Changes in soap flow rates yield opposite effects on the working regime to that of air

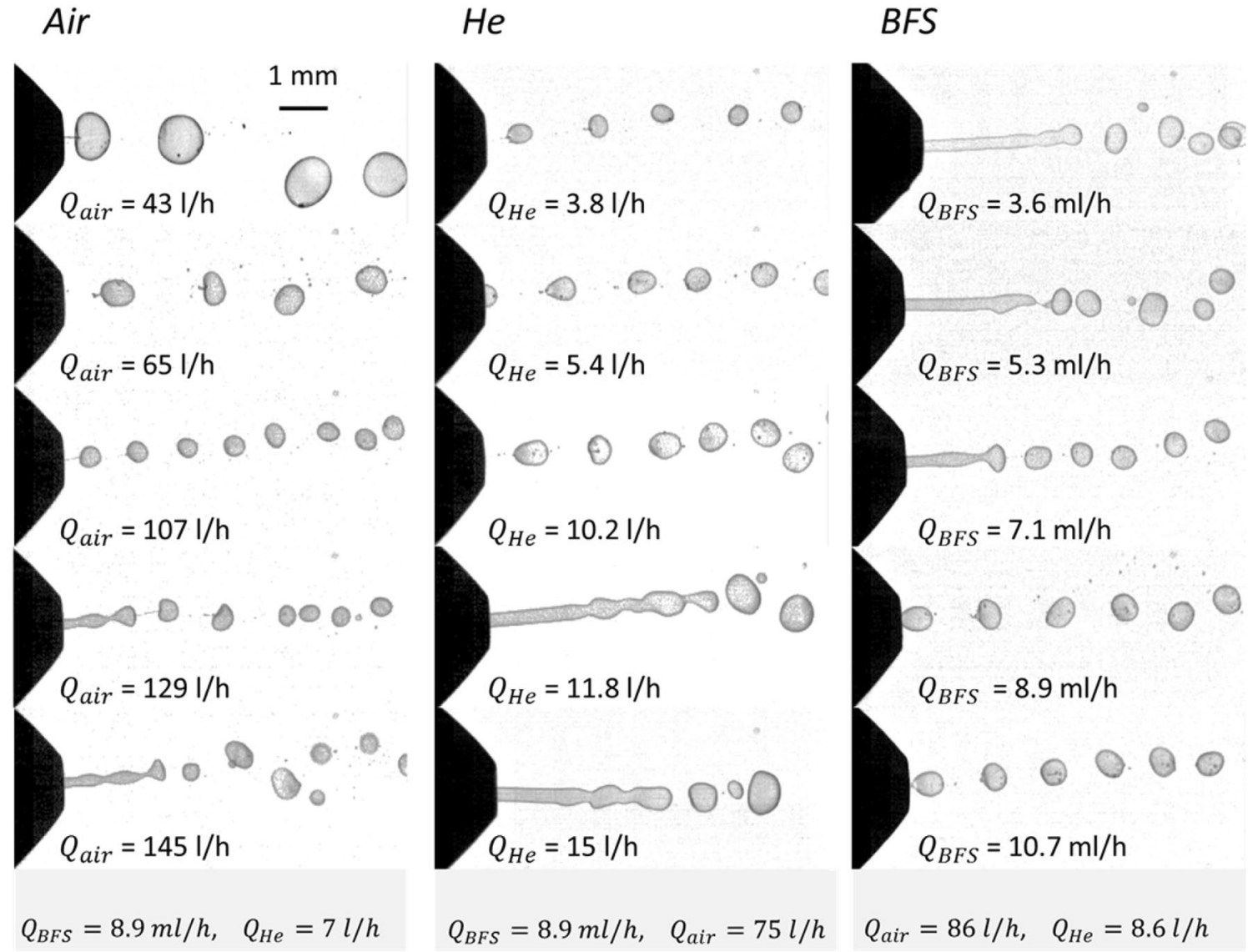

Fig. 8 Effect of flow rates on the bubble generation modes 
and helium. Low flow rates of soap are likely to yield jetting regime. By increasing the soap flow rate, the bubble production becomes less disperse and eventually transitions to bubbling $\left(Q_{\mathrm{BFS}}=8.9 \mathrm{ml} / \mathrm{h}\right)$. Moreover, changes in soap flow rates do not seem to affect either the bubble size or the frequency of bubble formation.

\subsection{Production regime envelopes}

A systematic procedure is implemented to map the production regimes. First, the volume flow rates of soap and helium are fixed while $Q_{\text {air }}$ is increased from the first working condition till beyond the transition point from bubbling to jetting. This process is repeated for different helium and soap flow rates until a complete mapping of the production regimes is obtained. The resulting mappings (Fig. 9) are given at fixed values of $Q_{\mathrm{BFS}}$.

For low values of $Q_{\mathrm{BFS}}$, the bubbling region is fairly limited. For higher values of $Q_{\mathrm{BFS}}$, the range of air and helium flow rates yielding bubbling regime increases significantly. The previous observation that jetting is present for high gas flow rates and low BFS flow rates (Sect. 5.1) is clearly shown in these maps. Moreover, it is observed that polydisperse bubbling occurs mostly for low air flow rates and can be easily avoided through increasing the air flow rate. The intersection of the neutral buoyancy condition $\left(Q_{\mathrm{He}}=900 Q_{\mathrm{BFS}}\right)$, represented by vertical black lines, with the bubbling range shows the desired working region. Imposing simultaneously these two criteria significantly restricts the choice of flow rates.

Although from the maps it appears that increasing soap flow rates is always beneficial, a more frequent occurrence of intermittent bubble production has been observed for $Q_{\mathrm{BFS}}$ $\geq 11 \mathrm{ml} / \mathrm{h}$ at high air volume flow rates, i.e., the bubble generator works only a fraction of the time, reducing the bubble production rate and increasing soap spitting. If the flow rates of soap are increased even further, the annular jet collapses and the bubble production ceases completely.

\section{Variance of time response and diameter}

The measurements of HFSB time response and diameter (from the glare points distance) are combined with the measurements of the shadow visualization at the same volume flow rates. In this way, the correlations between bubble size and time response dispersions to the production regimes are established. For this evaluation, the variance coefficient of the bubble diameter is introduced

$\mathrm{CV}_{\mathrm{d}}=\frac{\sigma_{\mathrm{d}}}{\left\langle d_{\mathrm{b}}\right\rangle}$,

where $\sigma_{\mathrm{d}}$ and $\left\langle d_{\mathrm{b}}\right\rangle$ are the standard deviation and average of the HFSB diameter, respectively.
The variance coefficient of HFSB diameter is analyzed from the PIV measurements, performed for 35 combinations of $Q_{\mathrm{air}}, Q_{\mathrm{He}}$ and $Q_{\mathrm{BFS}}$, as shown in Fig. 10. In comparison to the shadow visualization technique, the latter method does not suffer from nozzle exit effects and more bubble images were recorded, leading to a better convergence of $\sigma_{\mathrm{d}}$. From the shadow visualization, it was observed that the bubble generator was operating in the bubbling regime for 23 cases, in the jetting regime for 11 cases, and in one case double-bubbling occurred. When working in the bubbling regime small variations of bubble diameter were observed, with $\mathrm{CV}_{\mathrm{d}}$ between 2 and $3 \%$, with exception of three cases of low helium volume flow rates $\left(Q_{\mathrm{He}}=3.5 \mathrm{l} / \mathrm{h}\right)$, in which it was approximately $6 \%$. In the jetting or double bubbling regime, $\mathrm{CV}_{\mathrm{d}}$ was about $13 \%$. The consistency of the results shows quantitatively that the dispersion of HFSB diameter indeed depends on the production regime.

For the particle time response, the variance is shown in physical units (time) as the coefficient loses significance when $\tau_{\mathrm{p}}$ approaches zero. The relation of $\sigma_{\tau}$ with bubble size and density (through the ratio $Q_{\mathrm{BFS}} / Q_{\mathrm{He}}$ ) is shown in Fig. 11. There is no strong correlation of $\sigma_{\tau}$ with $Q_{\mathrm{BFS}} / Q_{\mathrm{He}}$ or with $d_{\mathrm{b}}$, and the fluctuations of $\tau_{\mathrm{p}}$ appear to be in the order of $40 \mu \mathrm{s}$ irrespective of bubble size. Furthermore, no clear difference in the time response dispersion is observed between bubbles produced in either bubbling or jetting regimes. In conclusion, neither the average bubble size nor the average density nor the dispersity of the bubble size influences significantly the fluctuations of time response. It is hypothesized that the observed dispersion of the time response is mainly a result of variations of the soap film thickness, leading to variations of bubble density.

Additionally, the streamwise velocity fluctuation $u_{\mathrm{rms}}^{\prime}$ measured with our reference DEHS was of $0.1 \mathrm{~m} / \mathrm{s}$. In a flow of $7000 \mathrm{~m} / \mathrm{s}^{2}$ deceleration, this implies that even a population of ideal tracers would exhibit a minimum $\sigma_{\tau}$ of $15 \mu \mathrm{s}$.

\section{Bubble production rate and size}

The inverse proportionality of the bubble volume and the production rate of bubbles for a monodisperse production of bubbles (bubbling regime) is confirmed experimentally (Fig. 12) for the NLR $\left(d_{0}=1 \mathrm{~mm}\right)$ and DLR $\left(d_{0}=0.75,1.00\right.$ and $1.50 \mathrm{~mm}$ ) bubble generators. The good agreement of the data indicates that Eq. (4) holds independently of bubble generator geometry.

From the flow visualizations (Sect. 5.1), it was observed that an increase in $Q_{\text {air }}$ causes a reduction of bubble size and an increase of bubble production rate. It was also observed that $Q_{\text {air }}$ is the most relevant input parameter influencing the production rate. Analyzing $f$ as a function of independent variations of air, helium and soap 


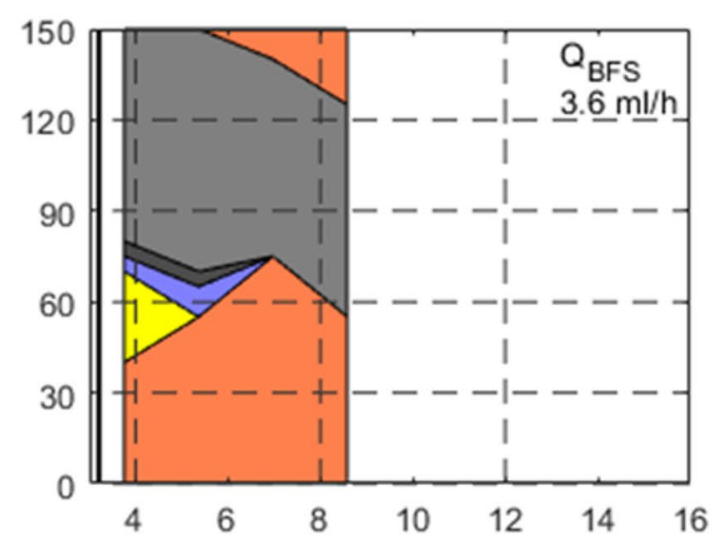

$$
\begin{array}{ll}
\square & \text { Not working } \\
\square & \text { Polydisperse-bubbling } \\
\square & \text { Bubbling } \\
\square & \text { Transition } \\
\square \quad \text { Jetting } \\
\hline
\end{array}
$$
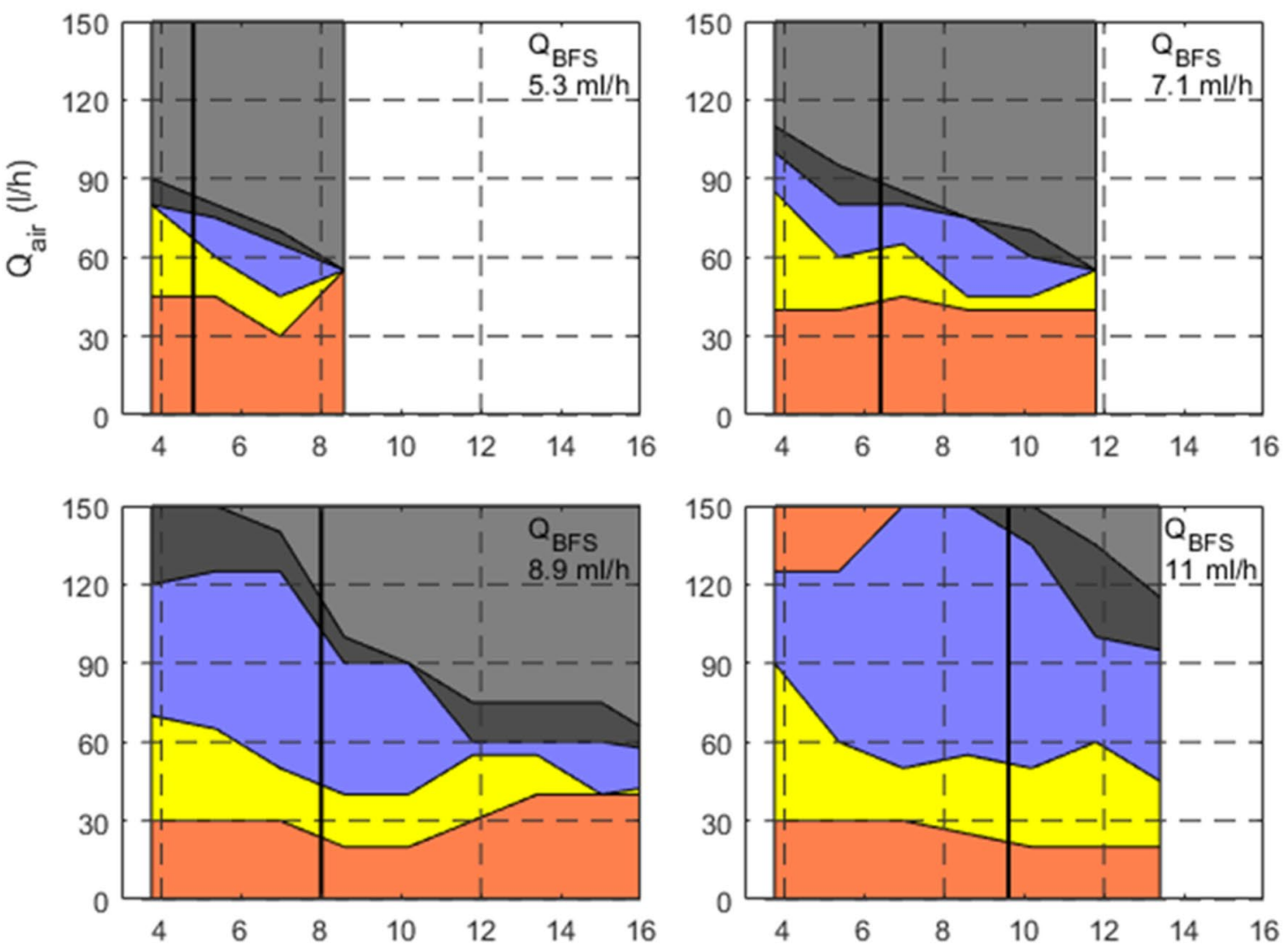

$Q_{\mathrm{He}}(\mathrm{l} / \mathrm{h})$

Fig. 9 Two-dimensional maps of production regimes for varying flow rates

flow rates (Fig. 13), it is observed that the production rate increases from 10 to $50 \mathrm{kHz}$ by increasing $Q_{\text {air }}$ from 40 to $120 \mathrm{l} / \mathrm{h}$, almost independently from the other flow rates. Moreover, a strong linear correlation of 0.91 is observed between $Q_{\text {air }}$ and $f$ for the measurements with the NLR generator in the bubbling regime (Fig. 14). The dependence of $f$ on $Q_{\text {air }}$ is attributed to the parameters governing annular jet instability (Shen and Li 1996). When $Q_{\text {air }}$ is increased, there is a reduction in the cross-section area of the helium-filled soap jet, combined with an increase in the jet velocity. The former enhances curvature effects, reducing therefore the wavelength of the fastest growing mode of instability, which determines the wavelength of bubble formation. A decrease in wavelength or an increase in jet velocity results in higher production rates (5). Moreover, the reason $Q_{\text {air }}$ is the main parameter concerning the HFSB production rate is that, within the available working conditions (bubbling regime, see Fig. 9), $Q_{\text {air }}$ has the 
Fig. 10 Variance coefficient of HFSB diameter for varying volume flow rates of air (65-160 1/h), helium (4-21 1/h) and soap $(3.5-11 \mathrm{ml} / \mathrm{h})$
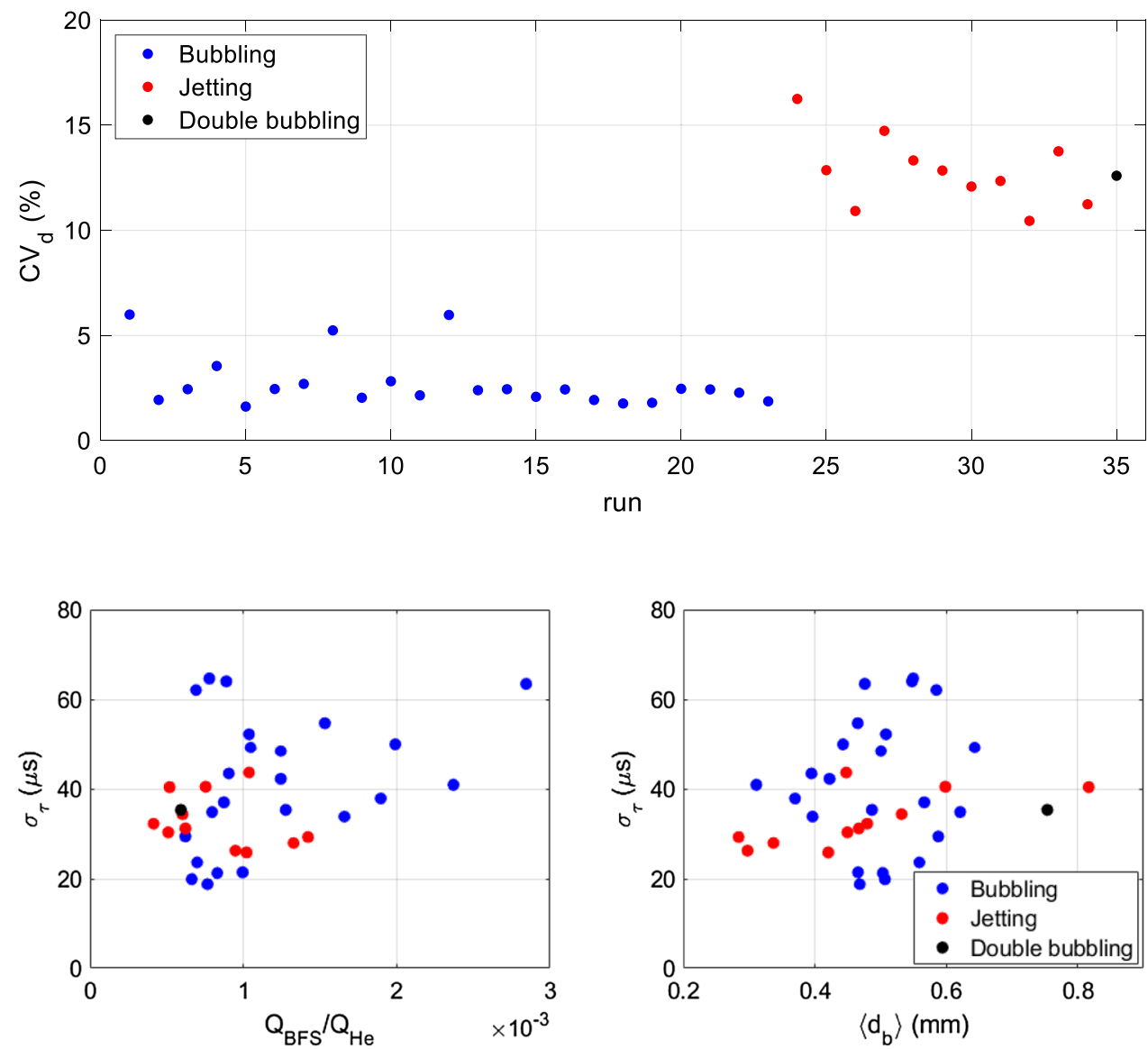

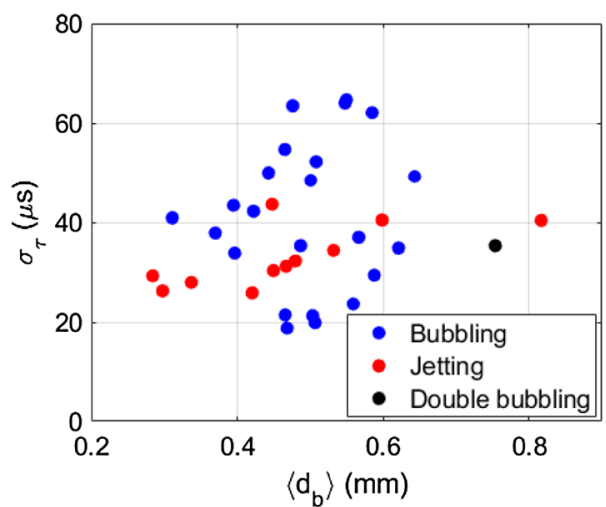

Fig. 11 Standard deviation of HFSB time response

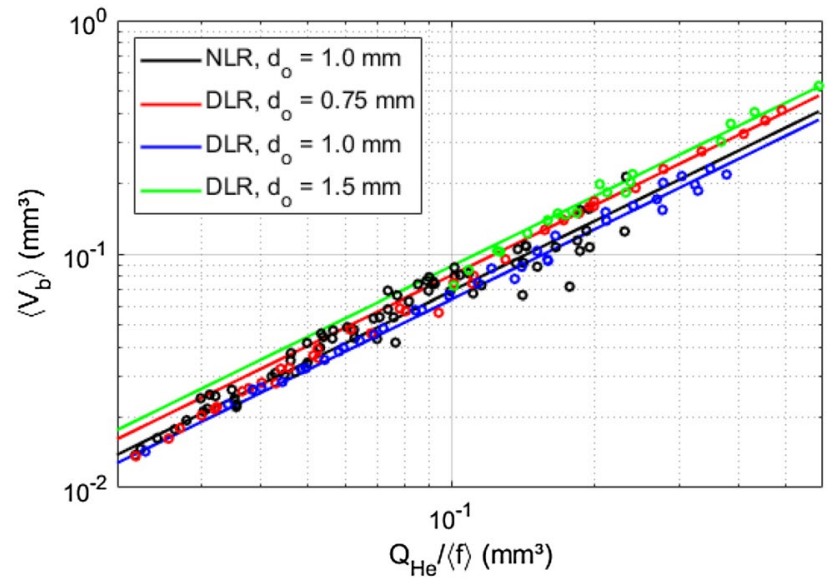

Fig. 12 Bubble volume vs. production rate

broadest available range (40-120 $\mathrm{l} / \mathrm{h})$ of this bubble generator design. In fact, increasing $Q_{\mathrm{BFS}}$ in $1 \mathrm{ml} / \mathrm{h}$ causes a decrease of about $2.5 \mathrm{kHz}$ in the production rate, while increasing $Q_{\mathrm{He}}$ in $1 \mathrm{l} / \mathrm{h}$ causes it to increase about $1.5 \mathrm{kHz}$. However, the available working conditions of $Q_{\mathrm{BFS}}$ and $Q_{\mathrm{He}}$ are quite restricted in comparison to that of air, ranging from 3 to $11 \mathrm{ml} / \mathrm{h}$ and from 4 to $16 \mathrm{l} / \mathrm{h}$, respectively. It should also be noted that when the focus is on producing neutrally buoyant bubbles, where $Q_{\mathrm{He}} / Q_{\mathrm{BFS}}$ must be kept constant, the effects of helium and soap on the production rate counteract each other.

A comparison of the HFSB production rate dependence on $Q_{\text {air }}$ obtained with different bubble generator geometries is shown in Fig. 14 (left). A linear relation between $f$ and $Q_{\text {air }}$ is observed for all configurations. The root-mean-square of the least square fits is about $4 \mathrm{kHz}$, which is attributed mainly to the influence of helium and soap. The production rate measured with the NLR generator shows a similar trend to that obtained with the DLR generator of smaller orifice size $(0.75 \mathrm{~mm})$. This can be explained by the phenomenon of vena contracta (Torricelli 1644; Reader-Harris 2015), where the effective orifice area is smaller than the geometric area. This effect is geometry dependent and it is conjectured that the resultant effective area of the NLR generator compares to effective area of the DLR generator of $0.75 \mathrm{~mm}$ orifice diameter. Furthermore, the data strongly indicate that when considering different orifice diameters for the same nozzle (DLR generator), the relation between $f$ and $Q_{\text {air }} / d_{\mathrm{o}}$ collapses into a single curve (Fig. 14, right).

The bubble velocity after detachment $u_{\mathrm{b}}$ (streamwise component), being proportional to the production rate (5), 

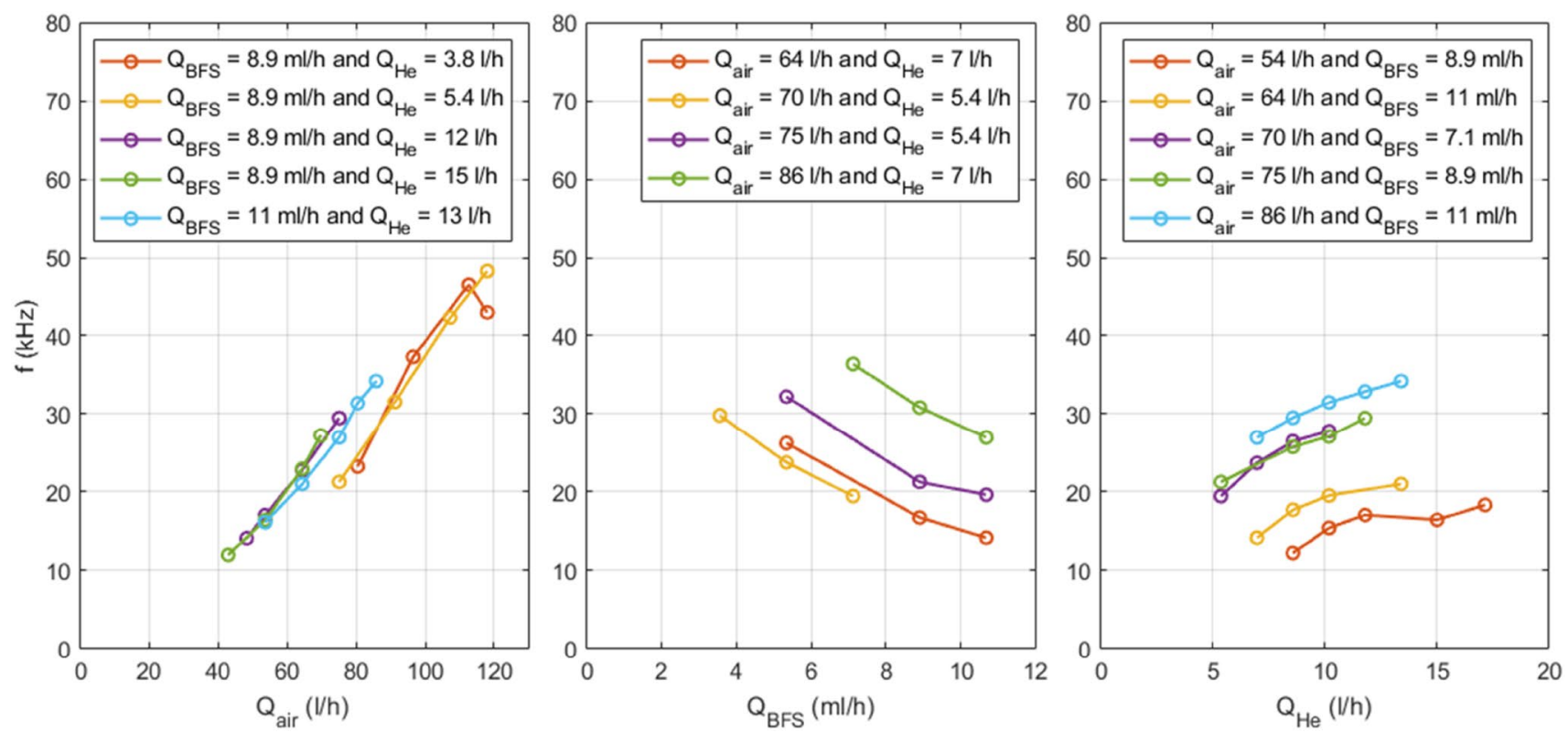

Fig. 13 Influence of air, helium and soap flow rates on the HFSB production rate

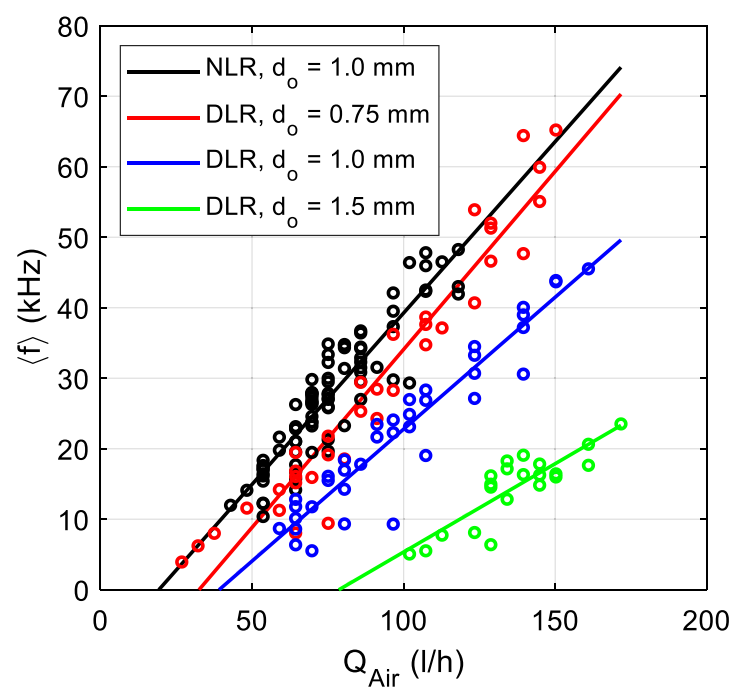

Fig. 14 Bubble production rate dependency on air flow rate. Helium flow rates varied in the range of $4-16 \mathrm{l} / \mathrm{h}$ for all generators, except for the DLR of $1.5 \mathrm{~mm}$ orifice diameter, for which it varied from 6

was also investigated (Fig. 15). It is seen that the HFSB velocity is typically between 20 and $40 \mathrm{~m} / \mathrm{s}$, demonstrating that in most cases, when the bubble generator is installed in the settling chamber, where the typical speed in subsonic wind tunnels is below $10 \mathrm{~m} / \mathrm{s}$, the external flow does not influence significantly the bubble production. The bubble velocity was expected to vary mainly with air velocity. At the orifice, the area of the annular cross section occupied by air was expected to scale with the orifice area $A_{\text {air }} \sim A_{\mathrm{o}}$ and the air velocity, therefore, to scale as $u_{\text {air }} \sim Q_{\text {air }} / d_{\mathrm{o}}^{2}$ (Fig. 15 ,

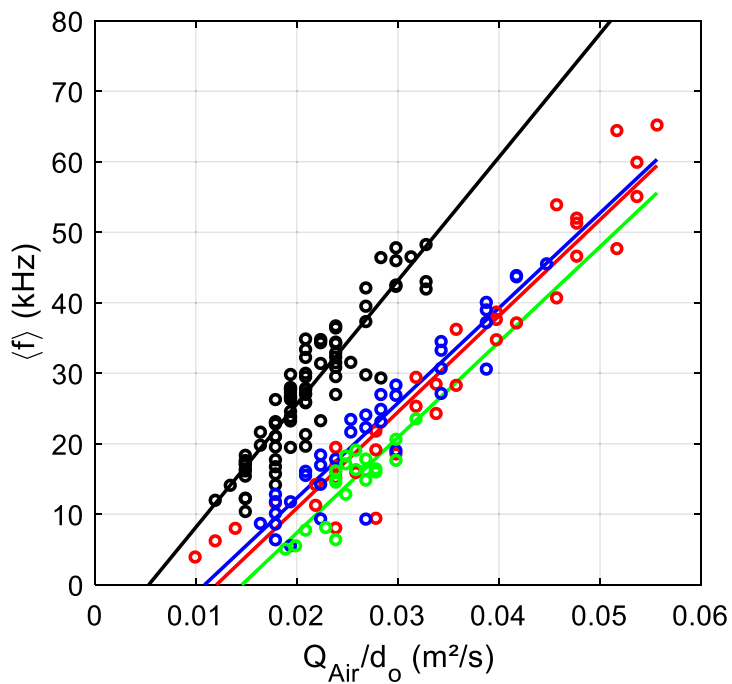

to $14 \mathrm{l} / \mathrm{h}$. Soap flow rates varied between 3 and $11 \mathrm{ml} / \mathrm{h}$ for the NLR generator and were kept constant at $8.9 \mathrm{ml} / \mathrm{h}$ for the DLR generators

left). Nevertheless, likewise the production rate, the measured bubble velocities for different orifice sizes show a better collapse with the ratio $Q_{\text {air }} / d_{\mathrm{o}}$ (Fig. 15, right). A complete analysis of the non-dimensional parameters governing this dependence is subject of further investigation.

Combining Eq. (4) with this empirical analysis, it is concluded that the bubble volume is proportional to the ratio of helium and air flow rates and to the orifice diameter. The bubble diameter, consequently, scales as: 

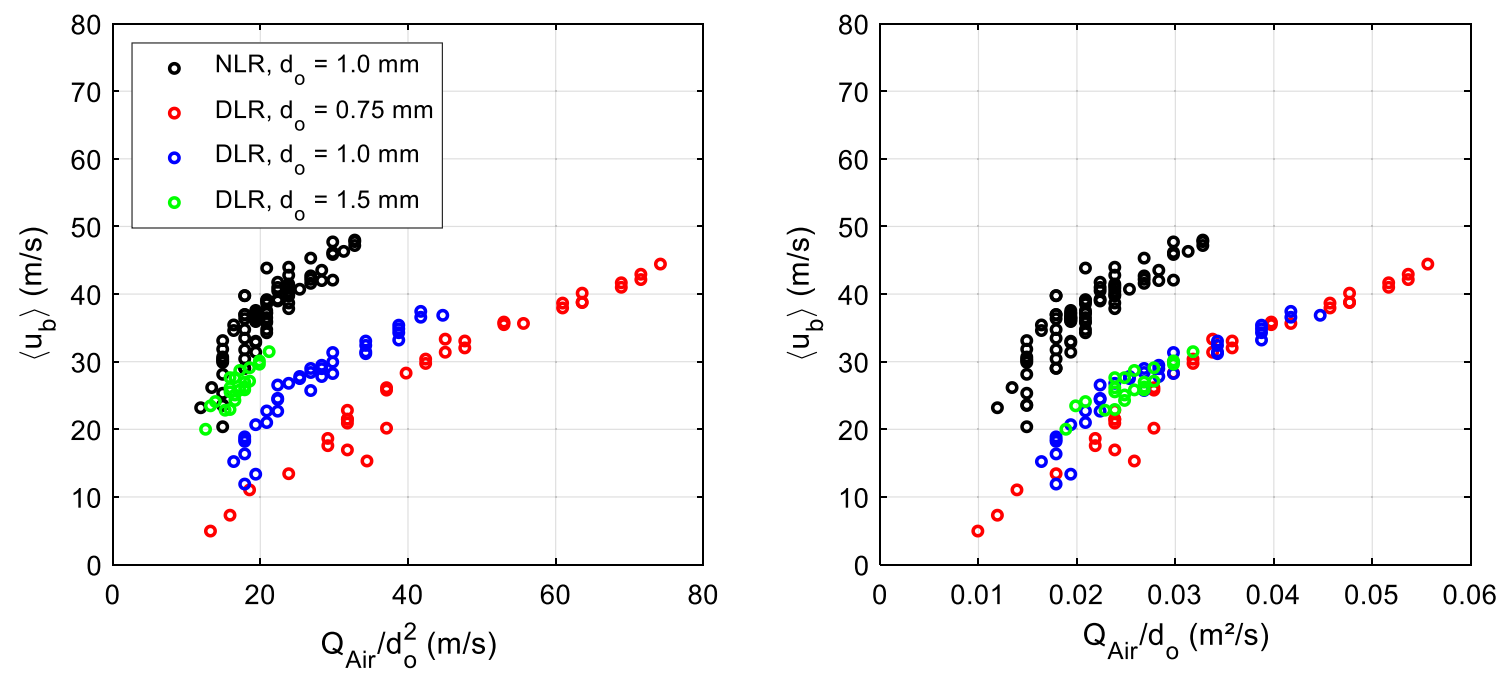

Fig. 15 Bubble velocity dependency on air volume flow rate

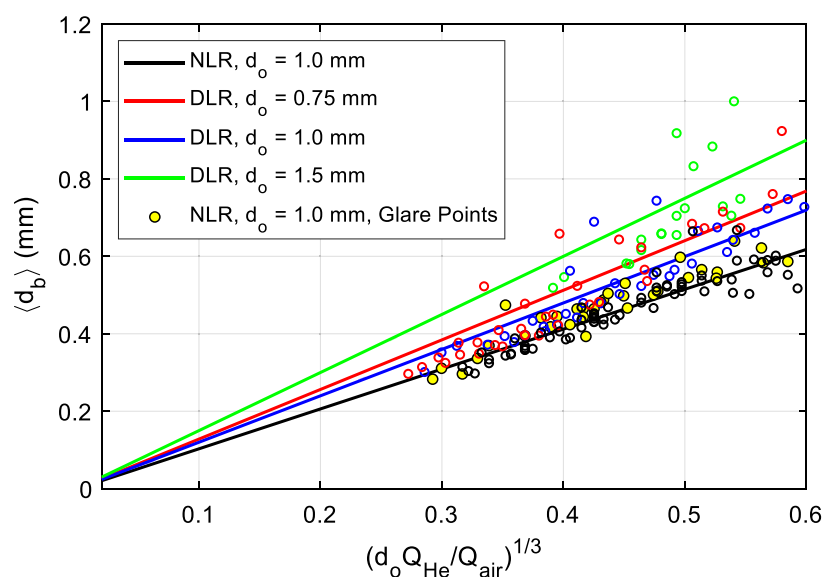

Fig. 16 HFSB diameter relation to the ratio of air and helium flow rates

$d_{\mathrm{b}} \sim\left(\frac{d_{\mathrm{o}} Q_{\mathrm{He}}}{Q_{\mathrm{air}}}\right)^{\frac{1}{3}}$.

The aforementioned proportionality is shown in Fig. 16. The data collapse well up to about $\left(d_{\mathrm{o}} Q_{\mathrm{He}} / Q_{\mathrm{air}}\right)^{1 / 3}=0.5$. For larger values, the data spread increases in particular for the DLR nozzles of 0.75 and $1.5 \mathrm{~mm}$ orifice diameters. For the $1.5 \mathrm{~mm}$ diameter case, the bubble generator needs high $Q_{\text {air }}$ to work properly since the orifice area has more than doubled in comparison to the reference $d_{\mathrm{o}}=1 \mathrm{~mm}$. Moreover, larger bubbles are more prone to deformation and they deviate from the spherical shape for a longer time after detachment, affecting the accuracy to which the bubble size is determined. Both the data from the cylinder experiment (bubble diameter estimation from glare points) and from shadow visualization are plotted together. No significant difference is observed between the two methods, demonstrating control and repeatability in both experiments. A direct comparison between ten data points obtained with both techniques with precisely the same flow rates showed that the shadow visualization measurements consistently underestimate the bubble size in $10 \%$ in comparison to the glare point method. The latter is deemed to be less prone to errors, since the bubbles are free from nozzle exit effects.

In conclusion, neglecting the small variations on the production rate due to $Q_{\mathrm{He}}$ and $Q_{\mathrm{BFS}}$, it is found that $f=f\left(Q_{\mathrm{air}}, d_{\mathrm{o}}\right)$ and $d_{\mathrm{b}}=d_{\mathrm{b}}\left(Q_{\mathrm{air}}, Q_{\mathrm{He}}, d_{\mathrm{o}}\right)$. From the perspective of PIV application, it is useful to summarize these results by showing frequency and bubble diameter together as a function of air and helium volume flow rate isolines, which is illustrated for the NLR nozzle ( $d_{\mathrm{o}}=1 \mathrm{~mm}$, Fig. 17). This diagram enables the selection of air and helium flow rates for a desired diameter and production rate. The optimal soap flow rate then follows from the neutral buoyancy relation $\left(Q_{\mathrm{He}} / Q_{\mathrm{BFS}}=900\right)$. Moreover, the region where the bubbles are simultaneously neutrally buoyant and monodisperse (produced in the bubbling regime), which has been determined from the flow visualizations (Fig. 9), is also included for completeness.

\section{Conclusions}

An experimental study is conducted to provide guidelines for generating monodisperse neutrally buoyant HFSB with chosen diameter and production rate. The above properties are achieved by properly selecting volume flow rates of air, helium and soap. The work finds its relevance from the 
Fig. 17 HFSB production rate and diameter as a function of air and helium flow rates. Production rate and bubble diameter dependency on the flow rates of air and helium were determined from the fitted curves to the NLR bubble generator data (Figs. 14 and 16). The ratio of neutral buoyancy was obtained from the measured time response (Fig. 5). The green region of monodisperse bubbles was obtained from the mapping of production regimes (Fig. 9)

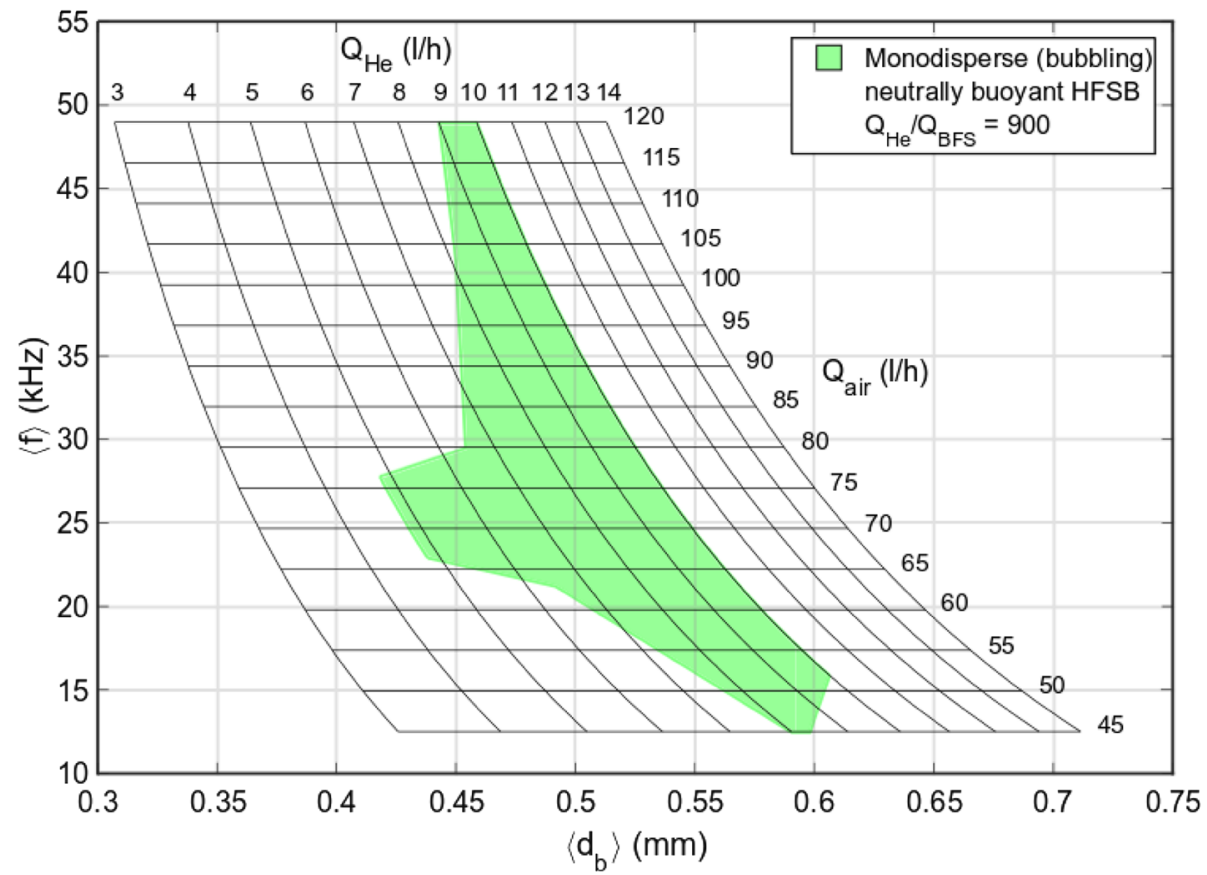

perspective of HFSB utilization as tracers for velocimetry techniques such as PIV and PTV.

The neutral buoyancy condition, which is essential for HFSB to behave as an ideal flow tracer, can be met by properly setting the helium and soap flow rates. Although a theoretical prediction yields $Q_{\mathrm{He}} / Q_{\mathrm{B} F S} \cong 1080$, it is found from experiments that the condition closest to neutral buoyancy is achieved when $Q_{\mathrm{He}} / Q_{\mathrm{BFS}}=900 \pm 50$. This indicates that a fraction of soap is spilled off in the form of droplets in the real process.

The HFSB time response was quantified in the laminar steady flow $\left(U_{\infty}=30 \mathrm{~m} / \mathrm{s}\right)$ in front of a cylinder of $50 \mathrm{~mm}$ diameter. This was found to be about 10-20 $\mu$ s when the bubble density is within $10 \%$ of the density of the surrounding air. For differences in the range of $10-20 \%$, the time response is within 20 and $50 \mu$ s. The time response dispersion $\sigma_{\tau}$ was quantified to be approximately $40 \mu \mathrm{s}$.

Several production regimes that depend on the combination of fluids flow rates were identified that can be classified into three main categories: polydisperse bubbling, bubbling and jetting. Polydisperse bubbling occurs mainly for low air flow rates and can be easily avoided. The production of HFSB in the bubbling regime is periodic and stable, resulting in the lowest variation of bubble size with a variance coefficient of bubble diameter $\mathrm{CV}_{\mathrm{d}}$ as small as $3 \%$. In the jetting regime, the generation of bubbles is more chaotic and the bubble size distribution is polydisperse with a typical $\mathrm{CV}_{\mathrm{d}}$ of $13 \%$. The dispersion of the time response, however, appears to be less dependent on the production regime. Transition from bubbling to jetting was found to occur when increasing the flow rates of air and helium or when decreasing the flow rate of soap. A synthesis of such conditions as a function of flow rates has been produced in the form of maps.

Furthermore, the results demonstrate that the bubble production rate in the bubbling regime increases linearly with the air volume flow rate and with the inverse of the orifice diameter $d_{\mathrm{o}}$, and that the bubble volume is proportional to the orifice size and the ratio of helium and air volume flow rates $V_{\mathrm{b}} \sim d_{\mathrm{o}} Q_{\mathrm{He}} / Q_{\mathrm{air}}$

Data availability The data points of figures of this article is available online at (https://doi.org/10.4121/uuid:7b0c9344-632c-422c-afdd48fdf39216fe).

Open Access This article is distributed under the terms of the Creative Commons Attribution 4.0 International License (http://creativeco mmons.org/licenses/by/4.0/), which permits unrestricted use, distribution, and reproduction in any medium, provided you give appropriate credit to the original author(s) and the source, provide a link to the Creative Commons license, and indicate if changes were made.

\section{References}

Adrian RJ, Westerweel J (2011) Particle image velocimetry. Cambridge University Press, New York

Bosbach J, Kühn M, Wagner C (2009) Large scale particle image velocimetry with helium filled soap bubbles. Exp Fluids 46:539

Caridi GCA (2018) Development and application of helium-filled soap bubbles for large-scale PIV experiments in aerodynamics. Ph.D. thesis 
Caridi GCA, Ragni D, Sciacchitano A, Scarano F (2016) HFSB seeding for large scale tomographic PIV in wind tunnels. Exp Fluids 57:190

Caridi GCA, Sciacchitano A, Scarano F (2017) Helium-filled soap bubbles for vortex core velocimetry. Exp Fluids 58:130

Clift R, Grace J, Webe M (1978) Bubbles, drops and particles. Academic Press, New York

Dehaeck S, van Beeck JPAJ, Riethmuller ML (2005) Extended glare point velocimetry and sizing for bubbly flows. Exp Fluids 39:407

Faleiros DE, Tuinstra M, Sciacchitano A, Scarano F (2018) Heliumfilled soap bubbles tracing fidelity in wall-bounded turbulence. Exp Fluids 59:56

Gibeau B, Ghaemi S (2018) A modular, 3D-printed helium-filled soap bubble generator for large-scale volumetric flow measurements. Exp Fluids 59:12

Jux C, Sciacchitano A, Schneiders JFG, Scarano F (2018) Robotic volumetric PIV of a full-scale cyclist. Exp in Fluids 59:74

Kähler C, Sammler B, Kompenhans J (2002) Generation and control of tracer particles for optical flow investigations in air. Exp Fluids 33:736

Kerho MF, Bragg MB (1994) Neutrally buoyant bubbles used as flow tracers in air. Exp Fluids 16:393

Kühn M, Bosbach J, Wagner C (2009) Experimental parametric study of forced and mixed convection in a passenger aircraft cabin mock-up. Build Environ 44:961-970

Kühn M, Ehrenfried K, Bosbach J, Wagner C (2011) Large-scale tomographic particle image velocimetry using helium-filled soap bubbles. Exp Fluids 50:929

Loth E (2008) Quasi-steady shape and drag of deformable bubbles and drops. Int J Multiph Flow 34(6):523-546

Mei R (1996) Velocity fidelity of flow tracer particles. Exp Fluids 22:1

Morias KLL, Caridi GCA, Sciacchitano A, Scarano F (2016) Statistical characterization of helium-filled soap bubbles tracing fidelity for
PIV. In: 18th International symposium on the application of laser and imaging techniques to fluid mechanics, Lisbon

Okuno Y, Fukuda T, Miwate Y, Kobayashi T (1993) Development of three dimensional air flow measuring method using soap bubbles. JSAE Rev 14(4):50-55

Pounder E (1956) Parachute inflation process. Wind-tunnel study WADC technical report 56-391, Equipment Laboratory of WrightPatterson Air Force Base, Ohio, USA, pp 17-18

Raffel M, Willert CE, Scarano F, Kähler C, Wereley ST, Kompenhans J (2018) Particle image velocimetry-a practical guide, 3rd edn. Springer, Berlin

Ragni D, Schrijer F, van Oudheusden BW, Scarano F (2011) Particle tracer response across shocks measured by PIV. Exp Fluids 50:53

Reader-Harris M (2015) Orifice plates and venturi tubes. Experimental fluid mechanics. Springer International Publishing, Cham

Scarano F, Ghaemi S, Caridi GCA, Bosbach J, Dierksheide U, Sciacchitano A (2015) On the use of helium filled soap bubbles for large scale tomographic PIV in wind tunnel experiments. Exp Fluids 56:42

Sciacchitano A, Giaquinta D, Schneiders JFG, Scarano F, van Rooijen BD, Funes DE (2018) Quantitative flow visualization of a turboprop aircraft by robotic volumetric velocimetry. In: 18th International symposium on flow visualization, Zurich

Shen J, Li X (1996) Instability of an annular viscous liquid jet. Acta Mech 114:167-183

Torricelli E (1644) Opera Geometrica, chap. De Motu Aquarum. Florentiae Typis Amotoris Massae et Laurentij de Landis

$\mathrm{Xu} \mathrm{H}$, Bodenschatz E (2008) Motion of inertial particles with size larger than Kolmogorov scale in turbulent flows. Phys D 237(14-17):2095-2100

Publisher's Note Springer Nature remains neutral with regard to jurisdictional claims in published maps and institutional affiliations. 